\title{
STUDIES OF KIDNEY FUNCTION IN CHILDREN
}

\section{Urea Clearance Values: (1) No Evidence of Kidney Disease (2) After Acute Hematuric Nephritis Following an Acute Infection}

(3) in the Acute Stage of Hematuric Nephritis ${ }^{1}$

\author{
By GLENN E. CULLEN, WALDO E. NELSON AND FREDERIC E. HOLMES \\ (From the Children's Hospital Research Foundation and the Department of Pediatrics, Uni- \\ versity of Cincinnati and the Children's Convalescent Home, Cincinnati)
}

(Received for publication May 6, 1935)

This study was made with three distinct purposes in view. The first was to determine whether the range and distribution of urea clearance values in children coincided with those in adults. The principles of the urea clearance test of renal function and its usefulness for adults have been adequately established by Möller, McIntosh and Van Slyke (1), and by other workers. There has been some question whether this test is as adequate for children as for adults.

The second purpose was to determine if it were possible by the urea clearance test to detect residual damage in the kidneys of children who had previously had acute hematuric nephritis. The general concensus of opinion is that, in the great majority of such cases, recovery of the kidney is complete. However, the urea clearance test has not been utilized in any extensive study of this problem.

The third purpose was to use the urea clearance test in children during the acute stage of hematuric nephritis, as an index of the degree of kidney damage, and to ascertain its usefulness during the convalescent stage in determining the rate at which the kidneys return to normal function.

\section{DETAILS OF THE EXAMINATIONS}

The control group. The 62 children in this group who were residents at the Children's Convalescent Home, had no evidence of kidney damage and no history of kidney disease. The groups included children who were primarily nutritional problems, children with some type of chronic heart disease, and children convalescing from various other conditions. This group of 62 chil-

\footnotetext{
1 Presented in abstract before the Central Society for Clinical Research, Chicago, November 1934.
}

dren not only served as the control for the group who had a history of acute hematuric nephritis but also furnished data concerning the urea clearance values to be expected in abnormal conditions other than kidney disease.

The children were given a breakfast of fruit, cereal with milk and an additional glass of milk. They received no eggs, meat or coffee. Approximately an hour after breakfast they voided and drank one glass of water. At the end of one hour they again voided and this urine was collected. Following this a sample of blood was taken from a vein, and a second glass of water given. At the end of the second hour the final sample of urine was collected. Complete collection of the samples was insured. While each collection period was not exactly an hour, the time at which the sample was taken was carefully recorded. This is the routine procedure recommended by Möller, McIntosh and Van Slyke (1). In all the data presented here the term " test" indicates the average of these two successive clearance values. It is to be noted that, with few exceptions, blood was taken following the collection of the first sample of urine.

The urea determinations on both the blood and urine were made with the Van Slyke manometric apparatus, 0.2 cc. of blood being used. Most determinations were done in duplicate. All the clearance values were corrected on the basis of the height of the child by the factor from McIntosh, Möller and Van Slyke's (2) line diagram.

Certain details of the urea determination should be noted. It is not possible with the gasometric urease method to get satisfactory blank determinations on $\mathbf{0 . 2}$ cc. of pure urea solutions corresponding to blood urea concentrations. The values are always low. However, with blood, the $0.2 \mathrm{cc}$. technic checks satisfactorily against the aeration technic, using $3 \mathrm{cc}$. for the same blood. Without giving in detail the studies which were 
made in an attempt to explain this fact, our conclusion was that in the absence of proteins of the blood the minute amount of mercury dissolved inhibits urease action whereas with protein present the slight amount of mercury that may be dissolved combines with the protein and therefore does not effect the enzyme. A minor deviation from Van Slyke's directions consisted in allowing the urease solution to act on the blood for at least two minutes, in order to insure complete transformation of the urea into ammonium carbonate. However, the possibility of significant ammonia formation from other nitrogen constituents of the blood was obviated by never allowing the urease to act more than 4 minutes. With this method, blanks on the urease solution should be run practically each day. "Double strength" (Squibb) urease powder should be used. Urease powder that has aged for several months is more satisfactory than fresh powder since it gives a lower and more constant "blank" value.

The group with a history of acute hematuric nephritis. Children who had had hematuric nephritis following an acute infection were brought to the clinic of the Children's Hospital for examination. $^{2}$ To avoid the factor of selection in this group an attempt was made to examine all children who had had acute nephritis within the past ten years and had been patients at the Children's Hospital or the Cincinnati General Hospital. For obvious reasons many of these children could not be returned for examination. However, it is probable that parents of children, who had had any persistent illnesses following their hospitalization, would be more willing to have them returned, and it is likely, therefore, that this group would include the greater proportion of children with permanently damaged kidneys, if such damage existed. Instructions were given that the children should receive the same breakfast outlined for the control group, and we believe that this procedure was followed in most instances. The children reported to the Clinic approximately an hour after breakfast. Clearance tests were then performed as in the control group. An interim history was taken, and a complete examination was made, which included blood pressure readings and an analysis of the urine for albumin, sugar, specific gravity, $\mathrm{pH}$ and microscopic study. In most instances the children appeared to be undisturbed by the examinations.

2 In this task we had the efficient cooperation of the Social Service Department of the Children's Hospital.

\section{RECORDING AND ANALYSIS OF DATA}

A large amount of accessory data were accumulated which cannot be reported here. Unless an exception is stated either in the tables or in the discussion, the physical and urine examinations and the history indicate that the child's condition was essentially normal.

The recording of data relating to children with a history of acute hematuric nephritis requires some explanation. In many cases, either because of an apparently unusual difference between the two successive clearance values of one test or because of some question concerning the clinical condition, the child returned to the clinic one or more times. In only two cases did these repeated tests vary significantly from each other. One of these is Number 5 of Table IV, who apparently showed a delayed recovery. The other (Case 92) was a girl so extremely apprehensive that three determinations had to be discarded. On the fourth visit to the clinic the disturbing psychic factors were eliminated, and the examination completed. In addition, several of the children first studied have been brought back after one or two years for further examinations.

The following arbitrary selection of data has been made. In Table II the last test made on the child is recorded. The histogram for this group (Figure 1) showing the frequency of occurrence of urea clearance test values is, therefore, based on the number of children rather than the number of determinations.

The number of children is sufficient to allow a biometric analysis of several secondary questions, such as the relation of clearance to age, to sex and to the time elapsed since the acute condition. For these analyses the repeated tests on any given. child are actually new data and are so used. For example, a child who had acute hematuric nephritis at $101 / 4$ years was studied two years ago at the age of 11 years. He has been studied twice since at $11 \frac{1}{2}$ and $121 / 2$ years of age respectively. The last determination at $121 / 2$ years is used for Table II and Figure 1. The first analysis is used for the analysis of "time since cessation of acute symptoms" in the " 1 month to 1 year" group and the average of the last two determinations for the corresponding group " 1 to 5 years." For the analysis of the influence of age, the first two 


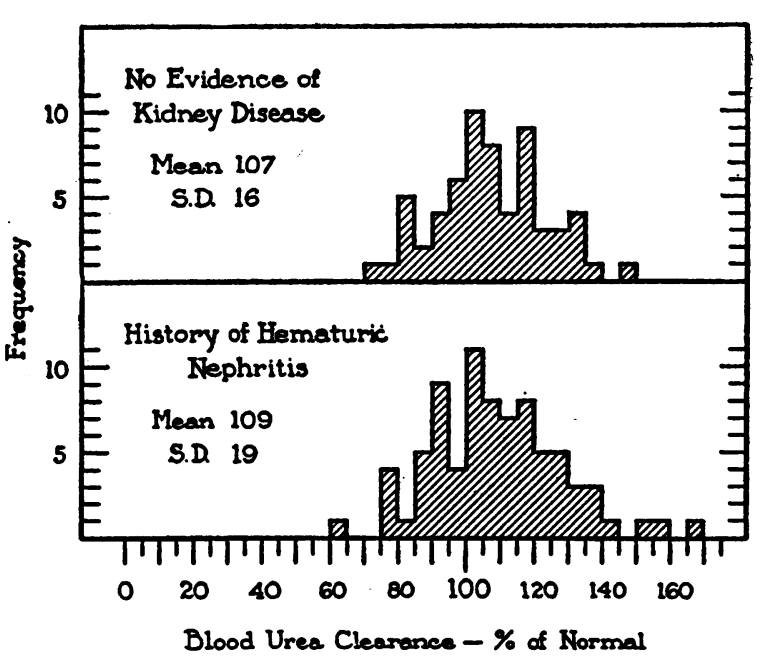

Fig. 1. Distribution Histograms of Urea Clearance Values in 62 Children with No Evidence of Kidney Disease, Upper Histogram, aNd in 78 Children Who Had Had Hematuric Nephritis Following an Acute Infection, Lower Histogram.

Frequencies are based on the number of children.

determinations are averaged for the " 8 to 12 year" group and the last determination used for the " 12 to 16 year" group.

Influence of types of clearance. There must be considered the comparative significance of the two different types of urea clearance, namely, " maximum " or "standard" $\left(C_{m}\right.$ or $\left.C_{8}\right)$. In accordance with the practice of Van Slyke, $C_{m}$ has been calculated for corrected urine volumes in excess of 2 cc. per minute, $C_{8}$ for those under 2 cc. Some workers have insisted that "maximum" clearances are more reliable than "standard" clearances. Analysis of the present data from the control group shows that if only the first hour clearances are considered (the first hour only because there are not enough $C_{8}$ values in the second hour) there are 47 "maximum" values with a mean of 112.1 , and 15 "standard" values with a mean of 116.2. The evidence is that $C_{m}$ and $C_{8}$ values are entirely comparable.

When the combinations for the two hours of the "test" used in this study are considered it is found that in the control group there are $47 C_{m} C_{m}$ combinations (both hours maximum) with a mean of 107.5, and $14 C_{8} C_{m}$ combinations (standard first hour, maximum second hour) with a mean of 106.1. This indicates that determinations with either of these combinations are entirely comparable.

There is somewhat more variation in the data from the group with a history of hematuric nephritis. The 52 tests with two successive maximum values $\left(C_{m} C_{m}\right)$ have practically the same mean value as that of the control group. The 15 tests with the $C_{8} C_{m}$ combination have a mean of 117.8 , which appears higher but which is not significantly different biometrically from the $C_{m} C_{m}$ sub-group. The mean value for the small sub-group of two successive standard values $\left(C_{8} C_{8}\right)$ is significantly higher than the mean value for $C_{m} C_{m}$ of this same major group, but the number (9) is too small to allow accurate deduction. Furthermore, it happens that 7 of the 9 are boys who in this group, as is pointed out below, tend to have higher clearance values. There are not enough combinations of maximum clearance followed by standard $\left(C_{m} C_{8}\right)$ to permit analysis.

It is apparent that when a glass of water is taken at the beginning of each period most of the clearances will be " maximum," and that " standard" and "maximum" are entirely comparable.

The difference between the first and second periods is of interest. For the higher clearance values this difference probably indicates only the great flexibility of the normal kidney, but in the lower zone of the normal range it may become important. Our impression, gained from this study and from other observations in known nephritic conditions, is that any single "test" should be questioned before too much attention is paid to the low value whenever one clearance is below normal and the other is in the normal range and when the difference between the two successive hour periods is greater than 75 per cent of their average (see, for example, Table II, Cases 187 and 149 with average clearance values of 76 and 87 respectively). In general, when the kidney is permanently damaged to such a degree that the clearance values are below normal, this damage is reflected in the consistency of the successive two hour clearances. In the acute stage this general statement does not obtain, because of psychic disturbances in the sick child, because of the rapidly changing degrees of irritation in the kidney or because of poor cooperation in obtaining urine samples. This lack of cooperation can, of course, be eliminated by the use of a catheter, but the practise in this hospital is to discourage catheterization unless really necessary. Furthermore, this study was planned to evaluate the test under conditions commonly used in adults.

The control group. The pertinent data concerning the control group are given in Table I and 
TABLE I

Urea clearance values in children who had no evidence of kidney disease, arranged in order of increasing clearances

\begin{tabular}{|c|c|c|c|c|c|c|c|c|c|c|c|c|c|c|c|}
\hline \multirow{4}{*}{$\underset{\substack{\text { Sub- } \\
\text { ject } \\
\text { ber }}}{\text {. }}$} & \multirow{4}{*}{ Age } & \multirow{4}{*}{ Sex } & \multirow{4}{*}{ Height } & \multirow{4}{*}{ Weight } & \multicolumn{10}{|c|}{ Clearance $(C)$ data } & \multirow{4}{*}{$\begin{array}{l}\text { Reason for admittance to } \\
\text { convalescent home }\end{array}$} \\
\hline & & & & & \multirow{3}{*}{$\begin{array}{c}\text { Blood } \\
\text { urea }\end{array}$} & \multicolumn{4}{|c|}{ 1st period } & \multicolumn{4}{|c|}{ 2nd period } & \multirow{3}{*}{$\begin{array}{c}C \\
\text { aver- } \\
\text { age }\end{array}$} & \\
\hline & & & & & & \multicolumn{3}{|c|}{ Urine } & \multirow{2}{*}{$C_{1}$} & \multicolumn{3}{|c|}{ Urine } & \multirow{2}{*}{$C_{2}$} & & \\
\hline & & & & & & Urea & $\boldsymbol{V}$ & $V_{\text {cor. }}$ & & Urea & $\boldsymbol{V}$ & $V_{\text {cor. }}$ & & & \\
\hline $\begin{array}{r}14 \\
131 \\
49 \\
26 \\
143\end{array}$ & \begin{tabular}{|c|} 
years \\
\\
11 \\
11 \\
12 \\
11 \\
12
\end{tabular} & $\begin{array}{c}\mathrm{M} \\
\mathbf{F} \\
\mathrm{F} \\
\mathrm{M} \\
\mathrm{F}\end{array}$ & \begin{tabular}{|l|} 
inches \\
\\
54 \\
59 \\
60 \\
54.5 \\
58
\end{tabular} & \begin{tabular}{|c|} 
pounds \\
59 \\
110 \\
86 \\
68 \\
73
\end{tabular} & $\begin{array}{c}\text { mgm. } \\
\text { per } \\
100 \\
c c . \\
25.8 \\
22.0 \\
24.4 \\
24.7 \\
28.5\end{array}$ & $\begin{array}{c}\text { mgm. } \\
\text { per } \\
100 \\
c c . \\
984 \\
254 \\
459 \\
1297 \\
182\end{array}$ & \begin{tabular}{|c|}
$c c$. \\
per \\
min- \\
ute \\
1.16 \\
3.73 \\
2.67 \\
0.90 \\
7.80
\end{tabular} & \begin{tabular}{|c|}
$c c$. \\
per \\
min- \\
ute \\
1.79 \\
4.96 \\
3.42 \\
1.37 \\
10.69
\end{tabular} & $\begin{array}{r}\text { per } \\
\text { cent } \\
\text { nor- } \\
\text { mal } \\
95 \\
76 \\
86 \\
113 \\
91\end{array}$ & \begin{tabular}{|c|} 
mgm. \\
per \\
100 \\
$c c$. \\
276 \\
231 \\
235 \\
167 \\
291
\end{tabular} & \begin{tabular}{|c|}
$c c$. \\
per \\
min- \\
ute \\
2.12 \\
4.20 \\
4.75 \\
3.85 \\
3.97
\end{tabular} & $\begin{array}{c}c c . \\
\text { per } \\
\text { min- } \\
\text { ute } \\
3.26 \\
5.59 \\
6.08 \\
5.85 \\
5.44\end{array}$ & $\begin{array}{c}\text { per } \\
\text { cent } \\
\text { nor- } \\
\text { mal } \\
47 \\
78 \\
78 \\
53 \\
75\end{array}$ & $\begin{array}{c}\text { per } \\
\text { cent } \\
\text { nor- } \\
\text { mal } \\
71 \\
77 \\
82 \\
83 \\
83\end{array}$ & $\begin{array}{l}\text { Behavior } \\
\text { Osteochondritis def. juvenile } \\
\text { Malnutrition } \\
\text { Post-chorea } \\
\text { Malnutrition }\end{array}$ \\
\hline $\begin{array}{r}15 \\
25 \\
7\end{array}$ & $\begin{array}{l}11 \\
11 \\
12\end{array}$ & $\begin{array}{l}\mathbf{M} \\
\mathbf{M} \\
\mathbf{M}\end{array}$ & $\begin{array}{l}53 \\
53 \\
55.5\end{array}$ & $\begin{array}{l}62 \\
69 \\
66\end{array}$ & \begin{tabular}{l|}
25.8 \\
26.9 \\
26.7
\end{tabular} & $\begin{array}{l}788 \\
256 \\
343\end{array}$ & $\begin{array}{l}1.34 \\
4.38 \\
3.49\end{array}$ & $\begin{array}{l}2.10 \\
6.88 \\
5.13\end{array}$ & $\begin{array}{l}86 \\
87 \\
88\end{array}$ & $\begin{array}{l}354 \\
223 \\
376\end{array}$ & $\begin{array}{l}2.83 \\
4.65 \\
3.13\end{array}$ & $\begin{array}{l}4.44 \\
7.30 \\
4.60\end{array}$ & $\begin{array}{l}82 \\
81 \\
87\end{array}$ & $\begin{array}{l}84 \\
84 \\
88\end{array}$ & $\begin{array}{l}\text { Malnutrition } \\
\text { Rheumatic carditis } \\
\text { Rheumatic carditis, tuberculous } \\
\text { hip }\end{array}$ \\
\hline $\begin{array}{r}124 \\
22\end{array}$ & $\begin{array}{l}11 \\
12\end{array}$ & $\underset{\mathrm{M}}{\mathrm{F}}$ & $\begin{array}{l}57 \\
58\end{array}$ & $\begin{array}{l}68 \\
77\end{array}$ & $\begin{array}{l}27.0 \\
24.9\end{array}$ & $\begin{array}{r}2202 \\
537\end{array}$ & $\begin{array}{l}0.33 \\
2.50\end{array}$ & $\begin{array}{l}0.47 \\
3.45\end{array}$ & $\begin{array}{r}104 \\
99\end{array}$ & $\begin{array}{l}678 \\
273\end{array}$ & $\begin{array}{l}1.53 \\
4.17\end{array}$ & $\begin{array}{l}2.16 \\
5.75\end{array}$ & $\begin{array}{l}72 \\
84\end{array}$ & $\begin{array}{l}88 \\
92\end{array}$ & $\begin{array}{l}\text { Malnutrition } \\
\text { Chronic bronchitis }\end{array}$ \\
\hline $\begin{array}{r}11 \\
126 \\
140 \\
51 \\
133\end{array}$ & $\begin{array}{r}7 \\
7 \\
7 \\
8 \\
13\end{array}$ & $\begin{array}{c}\mathrm{M} \\
\mathbf{M} \\
\mathbf{F} \\
\mathbf{F} \\
\mathbf{M}\end{array}$ & $\begin{array}{l}45 \\
48 \\
48.5 \\
47 \\
59\end{array}$ & $\begin{array}{l}48 \\
48 \\
47 \\
54 \\
66\end{array}$ & $\begin{array}{l}30.9 \\
23.4 \\
39.2 \\
23.4 \\
33.7\end{array}$ & $\begin{array}{r}421 \\
205 \\
2570 \\
250 \\
370\end{array}$ & $\begin{array}{l}2.93 \\
4.33 \\
0.42 \\
3.43 \\
4.83\end{array}$ & $\begin{array}{l}6.04 \\
8.10 \\
0.77 \\
6.59 \\
6.47\end{array}$ & $\begin{array}{r}110 \\
95 \\
107 \\
94 \\
95\end{array}$ & $\begin{array}{l}200 \\
208 \\
316 \\
356 \\
605\end{array}$ & $\begin{array}{l}4.32 \\
4.17 \\
4.05 \\
2.50 \\
3.10\end{array}$ & $\begin{array}{l}9.00 \\
7.80 \\
7.41 \\
4.80 \\
4.15\end{array}$ & $\begin{array}{l}78 \\
92 \\
80 \\
97 \\
99\end{array}$ & $\begin{array}{l}94 \\
94 \\
94 \\
96 \\
97\end{array}$ & $\begin{array}{l}\text { Tuberculous hip } \\
\text { Chronic upper resp. inf. } \\
\text { Malnutrition } \\
\text { Congenital cardiac } \\
\text { Rheumatic carditis }\end{array}$ \\
\hline $\begin{array}{l}44 \\
29 \\
41 \\
19 \\
40\end{array}$ & $\begin{array}{r}11 \\
5 \\
8 \\
5 \\
11\end{array}$ & $\begin{array}{l}\mathbf{M} \\
\mathbf{M} \\
\mathbf{M} \\
\mathbf{M} \\
\mathbf{M}\end{array}$ & $\begin{array}{l}48 \\
42.5 \\
48 \\
44 \\
54\end{array}$ & $\begin{array}{l}45 \\
38 \\
46 \\
44 \\
60\end{array}$ & $\begin{array}{l}27.3 \\
21.9 \\
24.1 \\
23.0 \\
29.5\end{array}$ & $\begin{array}{l}796 \\
212 \\
409 \\
223 \\
245\end{array}$ & $\begin{array}{l}1.49 \\
3.23 \\
2.50 \\
3.82 \\
5.83\end{array}$ & $\begin{array}{l}2.82 \\
7.33 \\
4.60 \\
8.17 \\
8.98\end{array}$ & $\begin{array}{r}110 \\
94 \\
104 \\
106 \\
99\end{array}$ & $\begin{array}{l}139 \\
128 \\
192 \\
162 \\
330\end{array}$ & $\begin{array}{l}6.52 \\
5.58 \\
4.65 \\
4.59 \\
4.35\end{array}$ & $\begin{array}{r}12.32 \\
12.67 \\
8.56 \\
9.82 \\
6.70\end{array}$ & $\begin{array}{r}84 \\
98 \\
91 \\
92 \\
100\end{array}$ & $\begin{array}{r}97 \\
96 \\
98 \\
99 \\
100\end{array}$ & $\begin{array}{l}\text { Malnutrition } \\
\text { Malnutrition } \\
\text { Malnutrition } \\
\text { Chronic mastoid infection } \\
\text { Malnutrition }\end{array}$ \\
\hline $\begin{array}{r}35 \\
43 \\
45 \\
21 \\
145\end{array}$ & $\begin{array}{r}9 \\
8 \\
11 \\
11 \\
8\end{array}$ & $\begin{array}{l}\mathbf{M} \\
\mathbf{M} \\
\mathbf{M} \\
\mathbf{M} \\
\mathbf{M}\end{array}$ & $\begin{array}{l}51 \\
47 \\
50.5 \\
53 \\
54.5\end{array}$ & $\begin{array}{l}54 \\
46 \\
57 \\
58 \\
60\end{array}$ & $\begin{array}{l}21.4 \\
24.1 \\
19.4 \\
21.9 \\
23.0\end{array}$ & $\begin{array}{l}347 \\
369 \\
435 \\
239 \\
324\end{array}$ & $\begin{array}{l}2.67 \\
2.22 \\
2.23 \\
4.63 \\
3.47\end{array}$ & $\begin{array}{l}4.51 \\
4.33 \\
3.84 \\
7.27 \\
5.24\end{array}$ & $\begin{array}{r}97 \\
88 \\
115 \\
104 \\
99\end{array}$ & $\begin{array}{l}239 \\
132 \\
162 \\
193 \\
293\end{array}$ & $\begin{array}{l}4.23 \\
8.17 \\
4.67 \\
5.55 \\
4.17\end{array}$ & $\begin{array}{r}7.15 \\
15.93 \\
8.03 \\
8.71 \\
6.30\end{array}$ & $\begin{array}{r}107 \\
116 \\
89 \\
102 \\
107\end{array}$ & $\begin{array}{l}102 \\
102 \\
102 \\
103 \\
103\end{array}$ & $\begin{array}{l}\text { Behavior } \\
\text { Post-chorea } \\
\text { Malnutrition } \\
\text { Chronic bronchitis } \\
\text { Malnutrition }\end{array}$ \\
\hline $\begin{array}{r}120 \\
50 \\
138 \\
32-2 \\
119\end{array}$ & $\begin{array}{l}5 \\
9 \\
7 \\
9 \\
9\end{array}$ & $\begin{array}{l}\mathrm{F} \\
\mathbf{F} \\
\mathbf{F} \\
\mathbf{M}\end{array}$ & $\begin{array}{l}43.5 \\
47.5 \\
46 \\
50 \\
55.5\end{array}$ & $\begin{array}{l}45 \\
52 \\
52 \\
57 \\
68\end{array}$ & $\begin{array}{l}21.9 \\
22.9 \\
26.5 \\
23.4 \\
26.6\end{array}$ & $\begin{array}{r}282 \\
1035 \\
1273 \\
300 \\
717\end{array}$ & $\begin{array}{l}2.82 \\
0.82 \\
0.65 \\
3.68 \\
1.77\end{array}$ & $\begin{array}{l}6.20 \\
1.55 \\
1.31 \\
6.40 \\
2.60\end{array}$ & $\begin{array}{r}107 \\
104 \\
103 \\
109 \\
93\end{array}$ & $\begin{array}{l}135 \\
218 \\
206 \\
209 \\
307\end{array}$ & $\begin{array}{l}5.45 \\
4.25 \\
5.03 \\
4.78 \\
5.15\end{array}$ & $\begin{array}{r}11.99 \\
8.03 \\
10.11 \\
8.32 \\
7.57\end{array}$ & $\begin{array}{r}99 \\
102 \\
105 \\
99 \\
117\end{array}$ & $\begin{array}{l}103 \\
103 \\
104 \\
104 \\
105\end{array}$ & $\begin{array}{l}\text { Post lung abscess } \\
\text { Malnutrition } \\
\text { Post-chorea } \\
\text { Malnutrition } \\
\text { Behavior }\end{array}$ \\
\hline $\begin{array}{c}20-2 \\
13 \\
9 \\
58 \\
122-2\end{array}$ & $\begin{array}{r}7 \\
8 \\
9 \\
12 \\
8\end{array}$ & $\begin{array}{r}\mathbf{M} \\
\mathbf{M} \\
\mathbf{M} \\
\mathbf{F} \\
\mathbf{F}\end{array}$ & $\begin{array}{l}45 \\
46.5 \\
49 \\
53 \\
51.5\end{array}$ & $\begin{array}{l}39 \\
44 \\
47 \\
62 \\
56\end{array}$ & $\begin{array}{l}25.2 \\
17.4 \\
22.0 \\
17.3 \\
27.5\end{array}$ & $\begin{array}{r}1774 \\
236 \\
1333 \\
534 \\
297\end{array}$ & $\begin{array}{l}0.41 \\
2.97 \\
0.72 \\
1.73 \\
5.17\end{array}$ & $\begin{array}{l}0.87 \\
5.88 \\
1.31 \\
2.73 \\
8.63\end{array}$ & $\begin{array}{l}121 \\
106 \\
128 \\
112 \\
124\end{array}$ & $\begin{array}{l}590 \\
219 \\
182 \\
132 \\
236\end{array}$ & $\begin{array}{l}1.38 \\
3.28 \\
4.34 \\
6.35 \\
4.77\end{array}$ & $\begin{array}{r}2.90 \\
6.49 \\
7.90 \\
10.03 \\
7.97\end{array}$ & $\begin{array}{r}91 \\
109 \\
87 \\
102 \\
91\end{array}$ & $\begin{array}{l}106 \\
108 \\
108 \\
108 \\
108\end{array}$ & $\begin{array}{l}\text { Malnutrition } \\
\text { Post-pneumonia } \\
\text { Post-pneumonia } \\
\text { Rheumatic carditis } \\
\text { Post-rheumatic fever }\end{array}$ \\
\hline $\begin{array}{r}18 \\
6 \\
47 \\
27 \\
137\end{array}$ & $\begin{array}{r}11 \\
13 \\
12 \\
10 \\
7\end{array}$ & $\begin{array}{c}\mathbf{M} \\
\mathbf{M} \\
\mathbf{M} \\
\mathbf{F} \\
\mathbf{F}\end{array}$ & $\begin{array}{l}\mathbf{5 3 . 5} \\
\mathbf{5 4} \\
\mathbf{5 6} \\
\mathbf{5 4} \\
\mathbf{5 0}\end{array}$ & $\begin{array}{l}60 \\
70 \\
77 \\
71 \\
53\end{array}$ & $\begin{array}{l}29.0 \\
24.5 \\
20.1 \\
23.3 \\
30.3\end{array}$ & $\begin{array}{r}2157 \\
442 \\
293 \\
340 \\
453\end{array}$ & $\begin{array}{l}0.52 \\
3.03 \\
3.92 \\
3.78 \\
3.59\end{array}$ & $\begin{array}{l}0.81 \\
4.67 \\
5.72 \\
5.75 \\
6.25\end{array}$ & $\begin{array}{l}124 \\
112 \\
111 \\
112 \\
124\end{array}$ & $\begin{array}{l}257 \\
435 \\
295 \\
340 \\
293\end{array}$ & $\begin{array}{l}5.07 \\
2.87 \\
3.80 \\
3.62 \\
4.47\end{array}$ & $\begin{array}{l}7.91 \\
4.42 \\
5.55 \\
5.50 \\
7.78\end{array}$ & $\begin{array}{r}94 \\
105 \\
108 \\
107 \\
100\end{array}$ & $\begin{array}{l}109 \\
109 \\
110 \\
110 \\
112\end{array}$ & $\begin{array}{l}\text { Malnutrition } \\
\text { Rheumatic carditis } \\
\text { Post-chorea } \\
\text { Malnutrition } \\
\text { Malnutrition }\end{array}$ \\
\hline $\begin{array}{r}128 \\
135 \\
12 \\
125 \\
144\end{array}$ & $\begin{array}{r}6 \\
10 \\
9 \\
7 \\
6\end{array}$ & $\begin{array}{l}\mathbf{M} \\
\mathbf{F} \\
\mathbf{M} \\
\mathbf{M} \\
\mathbf{M}\end{array}$ & $\begin{array}{l}44 \\
52 \\
50 \\
46.5 \\
46\end{array}$ & $\begin{array}{l}38 \\
56 \\
59 \\
50 \\
42\end{array}$ & $\begin{array}{l}29.1 \\
21.0 \\
24.5 \\
29.1 \\
27.7\end{array}$ & $\begin{array}{r}893 \\
299 \\
700 \\
847 \\
1311\end{array}$ & $\begin{array}{l}1.30 \\
3.33 \\
1.77 \\
1.46 \\
0.73\end{array}$ & $\begin{array}{l}2.82 \\
5.53 \\
3.12 \\
2.88 \\
1.47\end{array}$ & $\begin{array}{l}116 \\
105 \\
119 \\
112 \\
106\end{array}$ & $\begin{array}{l}189 \\
185 \\
255 \\
247 \\
212\end{array}$ & $\begin{array}{l}5.73 \\
6.42 \\
4.55 \\
5.35 \\
6.12\end{array}$ & $\begin{array}{r}12.43 \\
10.66 \\
8.01 \\
10.54 \\
12.24\end{array}$ & $\begin{array}{l}108 \\
125 \\
111 \\
119 \\
125\end{array}$ & $\begin{array}{l}112 \\
115 \\
115 \\
116 \\
116\end{array}$ & $\begin{array}{l}\text { Malnutrition } \\
\text { Chronic otitis media } \\
\text { Rheumatic carditis } \\
\text { Malnutrition } \\
\text { Chronic otitis media }\end{array}$ \\
\hline $\begin{array}{r}57 \\
30 \\
46 \\
52 \\
136\end{array}$ & $\begin{array}{r}12 \\
10 \\
10 \\
9 \\
10\end{array}$ & $\begin{array}{c}\mathbf{F} \\
\mathbf{M} \\
\mathbf{M} \\
\mathbf{F} \\
\mathbf{F}\end{array}$ & $\begin{array}{l}62 \\
56 \\
48.5 \\
49 \\
56\end{array}$ & $\begin{array}{l}96 \\
78 \\
45 \\
53 \\
71\end{array}$ & $\begin{array}{l}16.3 \\
23.1 \\
19.6 \\
20.4 \\
23.3\end{array}$ & $\begin{array}{r}1033 \\
387 \\
254 \\
229 \\
2012\end{array}$ & $\begin{array}{l}1.17 \\
3.47 \\
4.22 \\
4.12 \\
0.33\end{array}$ & \begin{tabular}{l|}
1.43 \\
5.07 \\
7.76 \\
7.50 \\
0.48
\end{tabular} & $\begin{array}{l}141 \\
113 \\
134 \\
112 \\
111\end{array}$ & $\begin{array}{r}145 \\
248 \\
424 \\
302 \\
1396\end{array}$ & $\begin{array}{l}6.40 \\
5.86 \\
1.95 \\
3.50 \\
0.88\end{array}$ & $\begin{array}{l}7.81 \\
8.56 \\
3.59 \\
6.37 \\
1.28\end{array}$ & $\begin{array}{r}92 \\
123 \\
104 \\
126 \\
126\end{array}$ & $\begin{array}{l}117 \\
118 \\
119 \\
119 \\
119\end{array}$ & $\begin{array}{l}\text { Rheumatic carditis } \\
\text { Malnutrition } \\
\text { Malnutrition } \\
\text { Post-chorea, rheumatic carditis } \\
\text { Malnutrition }\end{array}$ \\
\hline
\end{tabular}


TABLE I (continued)

\begin{tabular}{|c|c|c|c|c|c|c|c|c|c|c|c|c|c|c|c|}
\hline \multirow{4}{*}{$\begin{array}{c}\text { Sub- } \\
\text { ject } \\
\text { num- } \\
\text { ber }\end{array}$} & \multirow{4}{*}{ Age } & \multirow{4}{*}{ Sex } & \multirow{4}{*}{ Height } & \multirow{4}{*}{ Weight } & \multicolumn{10}{|c|}{ Clearance $(C)$ data } & \multirow{4}{*}{$\begin{array}{l}\text { Reason for admittance to } \\
\text { convalescent home }\end{array}$} \\
\hline & & & & & \multirow{3}{*}{$\begin{array}{c}\text { Blood } \\
\text { urea }\end{array}$} & \multicolumn{4}{|c|}{ 1st period } & \multicolumn{4}{|c|}{ 2nd period } & \multirow{3}{*}{$\mid \begin{array}{c}c \\
\text { aver- } \\
\text { age }\end{array}$} & \\
\hline & & & & & & \multicolumn{3}{|c|}{ Urine } & \multirow{2}{*}{$C_{1}$} & \multicolumn{3}{|c|}{ Urine } & \multirow{2}{*}{$C_{2}$} & & \\
\hline & & & & & & Urea & $\boldsymbol{V}$ & $V_{\text {cor. }}$ & & Urea & $\boldsymbol{V}$ & $V_{\text {oor. }}$ & & & \\
\hline & years & & inches & pounds & \begin{tabular}{|c|}
$m g m$ \\
$p e r$ \\
100 \\
$c c$.
\end{tabular} & $\begin{array}{l}\text { mgm. } \\
\text { per } \\
100 \\
c c .\end{array}$ & $\begin{array}{c}c c . \\
\text { per } \\
\text { min- } \\
\text { ute }\end{array}$ & $\begin{array}{c}c c . \\
\text { per } \\
\text { min- } \\
\text { ute }\end{array}$ & $\begin{array}{l}\text { per } \\
\text { cent } \\
\text { nor- } \\
\text { mal }\end{array}$ & $\begin{array}{c}\text { mgm. } \\
\text { per } \\
100 \\
c c .\end{array}$ & $\mid \begin{array}{c}c c . \\
\text { per } \\
\text { min- } \\
\text { uite }\end{array}$ & $\begin{array}{c}c c . \\
\text { per } \\
\text { min- } \\
\text { une }\end{array}$ & $\begin{array}{l}\text { per } \\
\text { cent } \\
\text { nor- } \\
\text { mal }\end{array}$ & $\begin{array}{l}\text { per } \\
\text { cent } \\
\text { nor- } \\
\text { mal }\end{array}$ & \\
\hline 127 & 8 & $\mathbf{F}$ & 50.5 & 62 & $29.0 \mid 1$ & 1303 & 1.01 & 1.73 & 110 & 465 & 3.55 & 6.07 & 130 & 120 & Rheumatic carditis \\
\hline 42 & 8 & $\mathbf{M}$ & 47 & 52 & 22.6 & 205 & $\mathbf{5 . 5 5}$ & 10.66 & 129 & 170 & 5.75 & 11.04 & 111 & 120 & Malnutrition \\
\hline 139 & 12 & $\mathbf{F}$ & 57 & 79 & 24.9 & 521 & 3.48 & 4.91 & 137 & 319 & 4.39 & 6.19 & 105 & 121 & Malnutrition \\
\hline $10-2$ & 9 & $\mathbf{M}$ & 48 & 58 & 17.7 & 148 & 6.35 & 11.81 & 131 & 191 & 4.38 & 8.15 & 117 & 124 & Rheumatic carditis \\
\hline 53 & 12 & $\mathbf{F}$ & 53 & 71 & 23.1 & 339 & 4.25 & 6.89 & 135 & 248 & 5.05 & 8.18 & 117 & 126 & Rheumatic carditis \\
\hline 132 & 9 & $\mathbf{M}$ & 52 & 76 & 34.92 & 2522 & 0.59 & 0.97 & 132 & 481 & 3.98 & 6.53 & 120 & 126 & Post-chorea \\
\hline 38 & 6 & $\mathbf{M}$ & 45 & 43 & 21.4 & 233 & 4.20 & 8.78 & 127 & 220 & 4.55 & 9.51 & 130 & 129 & Tuberculous hip \\
\hline 16 & 6 & $\mathbf{M}$ & 40.5 & 33 & 19.9 & 596 & 1.63 & 3.93 & 157 & 122 & 5.25 & 12.65 & 103 & 130 & Tuberculous spine \\
\hline 123 & 10 & $\mathbf{M}$ & 57 & 67 & 23.2 & 1134 & 1.73 & 2.44 & 159 & 313 & 3.92 & 5.53 & 100 & 130 & Malnutrition \\
\hline 31 & 10 & $\mathbf{M}$ & 51.5 & 57 & 22.3 & 1520 & 0.74 & 1.23 & 141 & 290 & 4.19 & 6.96 & 120 & 131 & Malnutrition \\
\hline 24 & 12 & $\mathbf{M}$ & 51 & 53 & 19.1 & 310 & 4.23 & 7.19 & 156 & 143 & 7.17 & 12.19 & 121 & 139 & Rheumatic carditis \\
\hline 34 & 12 & $\mathbf{M}$ & 56.5 & 76 & 27.5 & 503 & 4.67 & 6.63 & 161 & 374 & 5.10 & 7.24 & 131 & 146 & Malnutrition \\
\hline
\end{tabular}

summarized to show the distribution of urea clearance values in the upper histogram of Figure 1. The mean clearance value for the entire group is 107.3. The values for the standard deviation indicate that the " universe" is stable. Since the average normal for adults is by definition 100 per cent (Van Slyke, et al.), it is evident that the mean of 107 for this group of children is not significantly different from that of adults.

Of the 62 children of this group, 27 were admitted to the Convalescent Home as malnutrition problems, 13 children with various types of cardiac lesions and 22 children convalescing from other conditions. The question, whether the condition of these children had any influence on the normality of their kidney function must be considered. When, for analytic study, they were divided in several groups, it was found that in none of the sub-groups did the means of the clearance values differ significantly from each other or from the mean of the entire group. Of the various sub-groupings possible there are presented those which seem most logical, i.e., (1) age, (2) sex, (3) cardiac condition, (4) activity, i.e., whether the patients were confined to bed or allowed limited or full activity. In all cases the values for the probable error of the mean indicate that the number of cases is sufficient to establish a stable mean value.
Several points are of interest. There is no significant difference in the clearance values on the basis of age or sex. In children with cardiac lesions, regardless of activity, there was no differerence in the mean value of clearances. There does not appear to be any difference between the mean clearance values of the sub-groups on different degrees of activity. This last observation agrees with the conclusion from Van Slyke's laboratory, based on studies of normal adults, that the clearance values are essentially the same at rest and under ordinary activity.

It should be stated that in 8 of the 62 children a second test was made. The duplicate results were entirely consistent.

After this work was completed Payne and Shukry (3) reported somewhat similar studies on 39 children comparable to our control group. They conclude that "the average for children is higher than that for the adult and also that the zone into which most cases fall is also greater ( 80 to 140 ) as against ( 80 to 120 ). Inasmuch as once the kidney has passed the minimal threshold it is regarded as normal, the upper limit has no great clinical interest, and since the lower limit coincides with the adult value, it has not been deemed advisable to use a different normal for children." It should be noted that the method of recording observations by Payne and Shukry was 
TABLE II

Urea clearance values in children who had acute hematuric nephritis following an acute infection

\begin{tabular}{|c|c|c|c|c|c|c|c|c|c|c|c|c|c|c|c|}
\hline \multirow{4}{*}{$\begin{array}{l}\text { Subject } \\
\text { number }\end{array}$} & \multirow{4}{*}{ Age } & \multirow{4}{*}{ Sex } & \multirow{4}{*}{ Height } & \multirow{4}{*}{ Weight } & \multirow{4}{*}{ T.S.A.S.* } & \multicolumn{10}{|c|}{ Clearance $(C)$ data } \\
\hline & & & & & & \multirow{3}{*}{$\begin{array}{c}\text { Blood } \\
\text { urea }\end{array}$} & \multicolumn{4}{|c|}{ 1st period } & \multicolumn{4}{|c|}{ 2nd period } & \multirow{3}{*}{$\underset{\text { average }}{C}$} \\
\hline & & & & & & & \multicolumn{3}{|c|}{ Urine } & \multirow{2}{*}{$C_{1}$} & \multicolumn{3}{|c|}{ Urine } & \multirow{2}{*}{$C_{2}$} & \\
\hline & & & & & & & Urea & $\mathbf{v}$ & Veor. $_{\text {cor }}$ & & Urea & $\mathbf{v}$ & $\mathrm{v}_{\text {eor. }}$ & & \\
\hline $\begin{array}{l}104-2 \\
187 \\
188 \\
80-2 \\
101\end{array}$ & $\begin{array}{r}\text { years } \\
18 \\
7 \\
10 \\
18 \\
8\end{array}$ & \begin{tabular}{|c|}
$\mathrm{F}$ \\
$\mathrm{M}$ \\
$\mathrm{F}$ \\
$\mathrm{F}$ \\
$\mathrm{F}$
\end{tabular} & \begin{tabular}{|l|} 
inches \\
\\
59 \\
44.5 \\
48 \\
62.5 \\
50
\end{tabular} & \begin{tabular}{|c|} 
pounds \\
90 \\
45 \\
58 \\
90 \\
50
\end{tabular} & $\begin{array}{c}\text { years, } \\
\text { months } \\
3-5 \\
2-7 \\
3-4 \\
7-0 \\
1-0\end{array}$ & $\begin{array}{r}\begin{array}{c}m g m . \\
\text { per } \\
100 \\
\text { cc. }\end{array} \\
25.8 \\
31.5 \\
21.4 \\
17.9 \\
12.5\end{array}$ & $\begin{array}{r}\text { mgm. } \\
\text { per } \\
100 \text { cc. } \\
258 \\
1378 \\
362 \\
245 \\
114\end{array}$ & $\begin{array}{c}c c . \\
\begin{array}{c}c . \\
\text { per } \\
\text { minute }\end{array} \\
3.51 \\
0.54 \\
1.90 \\
3.90 \\
3.79\end{array}$ & $\begin{array}{c}c c . \\
\text { per } \\
\text { minute } \\
4.42 \\
1.15 \\
3.50 \\
4.52 \\
6.59\end{array}$ & $\begin{array}{c}\text { per } \\
\text { cent } \\
\text { normal } \\
59 \\
87 \\
79 \\
84 \\
80\end{array}$ & 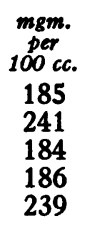 & $\begin{array}{c}c c . \\
\text { per } \\
\text { minute } \\
5.03 \\
2.97 \\
3.62 \\
4.47 \\
1.72\end{array}$ & $\begin{array}{c}c c . \\
p e r \\
\text { pinute } \\
6.33 \\
6.30 \\
6.66 \\
5.18 \\
2.99\end{array}$ & $\begin{array}{c}\text { per } \\
\begin{array}{c}\text { cent } \\
\text { normal }\end{array} \\
61 \\
64 \\
76 \\
72 \\
76\end{array}$ & $\begin{array}{c}\text { per } \\
\text { cent } \\
\text { normal } \\
60 \\
76 \\
78 \\
78 \\
78\end{array}$ \\
\hline $\begin{array}{c}88-3 \\
103 \\
90-2 \\
149 \\
97\end{array}$ & $\begin{array}{l}12 \\
10 \\
13 \\
18 \\
11\end{array}$ & $\begin{array}{c}\mathbf{F} \\
\mathbf{M} \\
\mathbf{F} \\
\mathbf{M} \\
\mathbf{F}\end{array}$ & $\begin{array}{l}64 \\
56 \\
52 \\
65 \\
54\end{array}$ & $\begin{array}{r}100 \\
64 \\
69 \\
116 \\
58\end{array}$ & $\begin{array}{l}1-10 \\
-11 \\
3-0 \\
6-0 \\
1-6\end{array}$ & $\begin{array}{l}20.0 \\
27.1 \\
20.4 \\
29.0 \\
16.9\end{array}$ & $\begin{array}{l}790 \\
205 \\
459 \\
900 \\
298\end{array}$ & $\begin{array}{l}1.32 \\
5.71 \\
1.82 \\
2.63 \\
2.58\end{array}$ & $\begin{array}{l}1.49 \\
8.34 \\
2.95 \\
2.84 \\
3.90\end{array}$ & $\begin{array}{r}89 \\
84 \\
88 \\
118 \\
92\end{array}$ & $\begin{array}{l}418 \\
335 \\
231 \\
442 \\
204\end{array}$ & $\begin{array}{l}2.43 \\
3.57 \\
3.45 \\
2.52 \\
3.45\end{array}$ & $\begin{array}{l}2.75 \\
5.21 \\
5.59 \\
2.72 \\
5.21\end{array}$ & $\begin{array}{l}76 \\
85 \\
84 \\
55 \\
84\end{array}$ & $\begin{array}{l}83 \\
85 \\
86 \\
87 \\
88\end{array}$ \\
\hline $\begin{array}{r}76 \\
186 \\
176 \\
82 \\
183\end{array}$ & $\begin{array}{r}7 \\
13 \\
14 \\
11 \\
11\end{array}$ & $\begin{array}{c}\mathbf{F} \\
\mathbf{M} \\
\mathbf{M} \\
\mathbf{M} \\
\mathbf{F}\end{array}$ & $\begin{array}{l}47 \\
61 \\
59.5 \\
58 \\
51\end{array}$ & $\begin{array}{r}53 \\
111 \\
88 \\
97 \\
54\end{array}$ & $\begin{array}{l}4-0 \\
2-6 \\
5-2 \\
6-0 \\
4-4\end{array}$ & $\begin{array}{l}23.9 \\
26.6 \\
20.2 \\
26.6 \\
22.6\end{array}$ & $\begin{array}{l}585 \\
620 \\
372 \\
935 \\
276\end{array}$ & $\begin{array}{l}1.53 \\
2.42 \\
2.77 \\
1.51 \\
3.63\end{array}$ & $\begin{array}{l}2.94 \\
3.00 \\
3.63 \\
2.10 \\
6.13\end{array}$ & $\begin{array}{r}96 \\
93 \\
89 \\
99 \\
100\end{array}$ & $\begin{array}{l}768 \\
644 \\
199 \\
325 \\
164\end{array}$ & $\begin{array}{l}0.98 \\
2.22 \\
5.45 \\
3.75 \\
5.08\end{array}$ & $\begin{array}{l}1.88 \\
2.75 \\
7.14 \\
5.21 \\
8.59\end{array}$ & $\begin{array}{l}81 \\
89 \\
93 \\
85 \\
83\end{array}$ & $\begin{array}{l}89 \\
91 \\
91 \\
92 \\
92\end{array}$ \\
\hline $\begin{array}{c}174 \\
59-2 \\
112 \\
196 \\
75-6\end{array}$ & $\begin{array}{r}9 \\
10 \\
9 \\
10 \\
9\end{array}$ & $\begin{array}{c}\mathbf{F} \\
\mathbf{M} \\
\mathbf{M} \\
\mathbf{F} \\
\mathbf{M}\end{array}$ & $\begin{array}{l}50 \\
53 \\
53 \\
50 \\
53\end{array}$ & $\begin{array}{l}52 \\
57 \\
65 \\
60 \\
68\end{array}$ & $\begin{array}{r}1-2 \\
-7 \\
3-8 \\
3-8 \\
1-0\end{array}$ & $\begin{array}{l}20.7 \\
30.2 \\
18.9 \\
19.9 \\
20.1\end{array}$ & $\begin{array}{l}250 \\
360 \\
529 \\
625 \\
215\end{array}$ & $\begin{array}{l}3.40 \\
3.96 \\
1.58 \\
1.47 \\
4.42\end{array}$ & $\begin{array}{l}5.92 \\
6.26 \\
2.53 \\
2.54 \\
6.94\end{array}$ & $\begin{array}{r}95 \\
100 \\
95 \\
107 \\
99\end{array}$ & $\begin{array}{l}186 \\
633 \\
458 \\
190 \\
200\end{array}$ & $\begin{array}{l}4.25 \\
1.93 \\
1.78 \\
3.67 \\
4.22\end{array}$ & $\begin{array}{l}7.40 \\
3.05 \\
2.85 \\
6.35 \\
6.63\end{array}$ & $\begin{array}{l}89 \\
85 \\
92 \\
81 \\
88\end{array}$ & $\begin{array}{l}92 \\
93 \\
94 \\
94 \\
94\end{array}$ \\
\hline $\begin{array}{c}92-4 \\
189 \\
94 \\
83 \\
102\end{array}$ & $\begin{array}{r}12 \\
8 \\
9 \\
9 \\
9\end{array}$ & $\begin{array}{c}\mathbf{F} \\
\mathbf{F} \\
\mathbf{M} \\
\mathbf{F} \\
\mathbf{M}\end{array}$ & $\begin{array}{l}57 \\
49.5 \\
48 \\
51 \\
50\end{array}$ & $\begin{array}{l}96 \\
59 \\
54 \\
54 \\
50\end{array}$ & $\begin{array}{l}2-0 \\
3-6 \\
1-4 \\
4-8 \\
1-3\end{array}$ & $\begin{array}{l}34.6 \\
21.7 \\
24.0 \\
25.1 \\
23.3\end{array}$ & $\begin{array}{l}753 \\
284 \\
428 \\
472 \\
303\end{array}$ & $\begin{array}{l}2.33 \\
3.47 \\
2.41 \\
2.57 \\
3.23\end{array}$ & $\begin{array}{l}3.26 \\
6.14 \\
4.53 \\
4.37 \\
5.68\end{array}$ & $\begin{array}{r}95 \\
107 \\
108 \\
109 \\
99\end{array}$ & $\begin{array}{l}329 \\
158 \\
290 \\
391 \\
238\end{array}$ & $\begin{array}{l}5.42 \\
4.98 \\
2.88 \\
2.45 \\
4.17\end{array}$ & $\begin{array}{l}7.59 \\
8.81 \\
5.41 \\
4.17 \\
7.34\end{array}$ & $\begin{array}{r}96 \\
85 \\
87 \\
87 \\
100\end{array}$ & $\begin{array}{r}96 \\
96 \\
98 \\
98 \\
100\end{array}$ \\
\hline $\begin{array}{c}151-2 \\
179-2 \\
177 \\
185 \\
89\end{array}$ & $\begin{array}{l}14 \\
14 \\
13 \\
18 \\
13\end{array}$ & $\begin{array}{l}\mathbf{M} \\
\mathbf{M} \\
\mathbf{M} \\
\mathbf{M} \\
\mathbf{F}\end{array}$ & $\begin{array}{l}56.5 \\
67.5 \\
56 \\
70.5 \\
58.5\end{array}$ & $\begin{array}{r}86 \\
129 \\
75 \\
144 \\
81\end{array}$ & $\begin{array}{l}3-0 \\
6-8 \\
4-6 \\
4-0 \\
5-0\end{array}$ & $\begin{array}{l}21.5 \\
31.2 \\
18.5 \\
19.5 \\
15.9\end{array}$ & $\begin{array}{r}614 \\
1694 \\
200 \\
505 \\
348\end{array}$ & $\begin{array}{l}1.73 \\
0.93 \\
4.52 \\
3.37 \\
2.66\end{array}$ & $\begin{array}{l}2.47 \\
0.96 \\
6.55 \\
3.17 \\
3.59\end{array}$ & $\begin{array}{r}94 \\
98 \\
95 \\
109 \\
105\end{array}$ & $\begin{array}{r}217 \\
1260 \\
230 \\
656 \\
196\end{array}$ & $\begin{array}{l}5.47 \\
1.78 \\
4.45 \\
2.18 \\
4.40\end{array}$ & $\begin{array}{l}7.82 \\
1.83 \\
6.45 \\
20.5 \\
5.94\end{array}$ & $\begin{array}{r}105 \\
102 \\
107 \\
92 \\
97\end{array}$ & $\begin{array}{l}100 \\
100 \\
101 \\
101 \\
101\end{array}$ \\
\hline $\begin{array}{c}99 \\
4-2 \\
63 \\
72-3 \\
69-3\end{array}$ & $\begin{array}{r}9 \\
6 \\
12 \\
9 \\
13\end{array}$ & $\begin{array}{l}\mathbf{F} \\
\mathbf{M} \\
\mathbf{M} \\
\mathbf{M} \\
\mathbf{M}\end{array}$ & $\begin{array}{l}51 \\
40.5 \\
55.5 \\
47 \\
66\end{array}$ & $\begin{array}{r}63 \\
43 \\
74 \\
46 \\
125\end{array}$ & $\begin{array}{l}1-1 \\
-7 \\
2-3 \\
1-3 \\
5-6\end{array}$ & $\begin{array}{l}29.4 \\
22.8 \\
25.2 \\
24.2 \\
25.8\end{array}$ & $\begin{array}{l}738 \\
229 \\
882 \\
391 \\
480\end{array}$ & $\begin{array}{l}2.10 \\
3.50 \\
1.38 \\
2.67 \\
3.65\end{array}$ & $\begin{array}{l}3.55 \\
7.74 \\
2.03 \\
5.18 \\
3.87\end{array}$ & $\begin{array}{r}119 \\
104 \\
95 \\
112 \\
96\end{array}$ & $\begin{array}{l}400 \\
164 \\
690 \\
544 \\
312\end{array}$ & $\begin{array}{l}2.78 \\
4.72 \\
2.07 \\
1.60 \\
6.50\end{array}$ & $\begin{array}{r}4.70 \\
10.43 \\
3.04 \\
3.10 \\
6.89\end{array}$ & $\begin{array}{r}85 \\
100 \\
111 \\
93 \\
111\end{array}$ & $\begin{array}{l}102 \\
102 \\
103 \\
103 \\
104\end{array}$ \\
\hline $\begin{array}{r}100 \\
194 \\
191 \\
73 \\
3\end{array}$ & $\begin{array}{c}4 \\
12 \\
11 \\
12- \\
9\end{array}$ & $\begin{array}{c}\mathbf{M} \\
\mathbf{F} \\
\mathbf{F} \\
\mathbf{M} \\
\mathbf{M}\end{array}$ & $\begin{array}{l}41 \\
56 \\
55 \\
59 \\
50\end{array}$ & $\begin{array}{r}39 \\
84 \\
95 \\
100 \\
62\end{array}$ & $\begin{array}{r}2-7 \\
4-0 \\
4-8 \\
3-0 \\
-1\end{array}$ & $\begin{array}{l}16.6 \\
20.1 \\
15.1 \\
32.0 \\
23.4\end{array}$ & $\begin{array}{r}126 \\
328 \\
870 \\
1582 \\
1993\end{array}$ & $\begin{array}{l}5.00 \\
3.18 \\
0.93 \\
1.29 \\
0.23\end{array}$ & \begin{tabular}{|r}
11.85 \\
4.64 \\
1.39 \\
1.70 \\
0.40
\end{tabular} & $\begin{array}{l}120 \\
101 \\
126 \\
120 \\
100\end{array}$ & $\begin{array}{r}185 \\
212 \\
293 \\
595 \\
1672\end{array}$ & $\begin{array}{l}2.62 \\
5.40 \\
2.28 \\
2.87 \\
0.45\end{array}$ & $\begin{array}{l}6.21 \\
7.88 \\
3.42 \\
3.79 \\
0.78\end{array}$ & $\begin{array}{r}92 \\
111 \\
88 \\
94 \\
116\end{array}$ & $\begin{array}{l}106 \\
106 \\
107 \\
107 \\
108\end{array}$ \\
\hline $\begin{array}{c}111 \\
98 \\
95 \\
5-3 \\
165\end{array}$ & $\begin{array}{c}11 \\
5 \\
16- \\
14 \\
9\end{array}$ & $\begin{array}{c}\mathbf{F} \\
\mathbf{M} \\
\mathbf{M} \\
\mathbf{F} \\
\mathbf{M}\end{array}$ & $\begin{array}{l}52 \\
41.5 \\
68 \\
60.5 \\
50\end{array}$ & $\begin{array}{r}59 \\
36 \\
143 \\
107 \\
57\end{array}$ & $\begin{array}{l}3-0 \\
1-2 \\
5-0 \\
1-8 \\
1-8\end{array}$ & $\begin{array}{l}21.9 \\
14.0 \\
22.0 \\
24.9 \\
25.1\end{array}$ & $\begin{array}{r}728 \\
275 \\
430 \\
1345 \\
1364\end{array}$ & $\begin{array}{l}1.57 \\
1.94 \\
4.27 \\
1.11 \\
0.81\end{array}$ & $\begin{array}{l}2.54 \\
4.52 \\
4.38 \\
1.41 \\
1.40\end{array}$ & $\begin{array}{l}113 \\
119 \\
114 \\
119 \\
119\end{array}$ & $\begin{array}{r}293 \\
100 \\
1092 \\
375 \\
190\end{array}$ & $\begin{array}{l}3.57 \\
4.48 \\
1.21 \\
3.93 \\
5.73\end{array}$ & $\begin{array}{r}5.78 \\
10.44 \\
1.24 \\
4.99 \\
9.97\end{array}$ & $\begin{array}{r}103 \\
99 \\
103 \\
100 \\
100\end{array}$ & $\begin{array}{l}108 \\
109 \\
109 \\
110 \\
110\end{array}$ \\
\hline $\begin{array}{c}110 \\
67 \\
74-9 \\
107 \\
85-2\end{array}$ & $\begin{array}{r}8 \\
10 \\
7 \\
4 \\
15\end{array}$ & $\begin{array}{c}\mathbf{F} \\
\mathbf{F} \\
\mathbf{M} \\
\mathbf{F} \\
\mathbf{F}\end{array}$ & $\begin{array}{l}48 \\
51 \\
51 \\
40.5 \\
63\end{array}$ & $\begin{array}{l}50 \\
60 \\
65 \\
35 \\
99\end{array}$ & $\begin{array}{c}2-11 \\
2-4 \\
-1.5 \\
-11 \\
1-3\end{array}$ & $\begin{array}{l}15.7 \\
25.0 \\
27.3 \\
28.0 \\
30.5\end{array}$ & $\begin{array}{r}605 \\
813 \\
573 \\
2005 \\
3359\end{array}$ & $\begin{array}{l}1.20 \\
1.67 \\
2.38 \\
0.43 \\
0.34\end{array}$ & $\begin{array}{l}2.26 \\
2.82 \\
4.24 \\
1.03 \\
0.40\end{array}$ & $\begin{array}{l}116 \\
122 \\
119 \\
135 \\
129\end{array}$ & $\begin{array}{r}162 \\
230 \\
242 \\
464 \\
1252\end{array}$ & $\begin{array}{l}4.00 \\
4.77 \\
5.00 \\
1.73 \\
1.40\end{array}$ & $\begin{array}{l}7.52 \\
8.06 \\
8.90 \\
4.15 \\
1.65\end{array}$ & $\begin{array}{r}104 \\
99 \\
105 \\
92 \\
98\end{array}$ & $\begin{array}{l}110 \\
111 \\
112 \\
114 \\
114\end{array}$ \\
\hline
\end{tabular}

* Time since acute symptoms. 
TABLE II (continued)

\begin{tabular}{|c|c|c|c|c|c|c|c|c|c|c|c|c|c|c|c|}
\hline \multirow{4}{*}{$\begin{array}{l}\text { Subject } \\
\text { number }\end{array}$} & \multirow{4}{*}{ Age } & \multirow{4}{*}{ Sex } & \multirow{4}{*}{ Height } & \multirow{4}{*}{ Weight } & \multirow{4}{*}{ T.S.A.S. ${ }^{*}$} & \multicolumn{10}{|c|}{ Clearance $(C)$ data } \\
\hline & & & & & & \multirow{3}{*}{$\begin{array}{c}\text { Blood } \\
\text { urea }\end{array}$} & \multicolumn{4}{|c|}{ 1st period } & \multicolumn{4}{|c|}{ 2nd period } & \multirow{3}{*}{$\underset{\text { average }}{C}$} \\
\hline & & & & & & & \multicolumn{3}{|c|}{ Urine } & \multirow{2}{*}{$C_{1}$} & \multicolumn{3}{|c|}{ Urine } & \multirow{2}{*}{$C_{3}$} & \\
\hline & & & & & & & Urea & $\mathbf{v}$ & Voor. & & Urea & $\mathbf{v}$ & $V_{\text {eor. }}$ & & \\
\hline $\begin{array}{c}91 \\
121-2 \\
71 \\
108 \\
105-2\end{array}$ & $\begin{array}{r}\text { years } \\
4 \\
8 \\
8 \\
12 \\
13\end{array}$ & $\begin{array}{c}\mathbf{F} \\
\mathbf{M} \\
\mathbf{F} \\
\mathbf{F} \\
\mathbf{M}\end{array}$ & \begin{tabular}{|l|} 
inches \\
\\
39 \\
48.5 \\
47 \\
58 \\
54
\end{tabular} & \begin{tabular}{|c} 
pounds \\
34 \\
56 \\
51 \\
75 \\
70
\end{tabular} & $\begin{array}{c}\text { years, } \\
\text { months } \\
1-8 \\
-2 \\
2-7 \\
5-4 \\
2-2\end{array}$ & $\begin{array}{c}\begin{array}{c}\text { mgm. } \\
\text { per } \\
100 \\
\text { cc. }\end{array} \\
23.9 \\
20.8 \\
21.3 \\
21.6 \\
17.4\end{array}$ & $\begin{array}{r}\text { mgm. } \\
\text { per } \\
100 \text { cc. } \\
2434 \\
340 \\
1790 \\
278 \\
570\end{array}$ & $\begin{array}{c}c c . \\
\text { per } \\
\text { minute } \\
0.26 \\
2.62 \\
0.33 \\
5.55 \\
1.77\end{array}$ & $\begin{array}{c}c c . \\
\text { per } \\
\text { minute } \\
0.65 \\
4.82 \\
0.64 \\
7.60 \\
2.73\end{array}$ & $\begin{array}{c}\text { per } \\
\text { cent } \\
\text { normal } \\
152 \\
105 \\
124 \\
130 \\
119\end{array}$ & 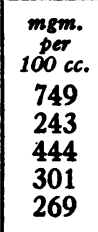 & $\begin{array}{c}c c . \\
\text { per } \\
\text { minute } \\
0.71 \\
4.42 \\
1.97 \\
3.98 \\
3.57\end{array}$ & $\begin{array}{c}c c . \\
\text { per } \\
\text { minute } \\
1.78 \\
8.13 \\
3.84 \\
5.45 \\
5.50\end{array}$ & \begin{tabular}{|c|} 
per \\
cent \\
normal \\
78 \\
127 \\
107 \\
101 \\
113
\end{tabular} & 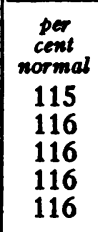 \\
\hline $\begin{array}{c}190 \\
166 \\
113-3 \\
64 \\
169\end{array}$ & $\begin{array}{r}7 \\
9 \\
12 \\
7 \\
9\end{array}$ & $\begin{array}{c}\mathbf{M} \\
\mathbf{F} \\
\mathbf{F} \\
\mathbf{M} \\
\mathbf{F}\end{array}$ & $\begin{array}{l}42.5 \\
50 \\
62 \\
45 \\
50\end{array}$ & $\begin{array}{l}43 \\
58 \\
95 \\
46 \\
50\end{array}$ & $\begin{array}{l}3-3 \\
1-3 \\
3-6 \\
1-8 \\
3-0\end{array}$ & $\begin{array}{l}17.7 \\
19.2 \\
15.4 \\
25.9 \\
16.8\end{array}$ & $\begin{array}{r}160 \\
355 \\
202 \\
1154 \\
456\end{array}$ & $\begin{array}{l}5.03 \\
2.82 \\
5.73 \\
0.95 \\
1.93\end{array}$ & \begin{tabular}{|r}
11.32 \\
4.91 \\
6.99 \\
2.00 \\
3.36
\end{tabular} & $\begin{array}{l}136 \\
121 \\
122 \\
119 \\
122\end{array}$ & $\begin{array}{r}98 \\
182 \\
245 \\
180 \\
166\end{array}$ & $\begin{array}{l}5.80 \\
5.10 \\
4.35 \\
6.22 \\
5.20\end{array}$ & \begin{tabular}{|r}
13.05 \\
8.87 \\
5.31 \\
13.06 \\
9.05
\end{tabular} & $\begin{array}{r}96 \\
112 \\
113 \\
121 \\
119\end{array}$ & $\begin{array}{l}116 \\
117 \\
118 \\
120 \\
121\end{array}$ \\
\hline $\begin{array}{r}93 \\
181 \\
197 \\
168 \\
66\end{array}$ & $\begin{array}{r}11 \\
17 \\
14 \\
5 \\
5\end{array}$ & $\begin{array}{c}\mathbf{M} \\
\mathbf{M} \\
\mathbf{M} \\
\mathrm{F} \\
\mathbf{F}\end{array}$ & $\begin{array}{l}60.5 \\
68.5 \\
63 \\
42.5 \\
44\end{array}$ & $\begin{array}{r}98 \\
142 \\
99 \\
40 \\
47\end{array}$ & $\begin{array}{l}1-6 \\
9-6 \\
8-8 \\
3-0 \\
1-0\end{array}$ & $\begin{array}{l}19.2 \\
24.5 \\
16.6 \\
16.7 \\
23.7\end{array}$ & $\begin{array}{r}1532 \\
1574 \\
1663 \\
400 \\
232\end{array}$ & $\begin{array}{l}0.55 \\
1.21 \\
0.49 \\
1.52 \\
4.65\end{array}$ & \begin{tabular}{|r}
0.70 \\
1.20 \\
0.57 \\
3.91 \\
10.14
\end{tabular} & $\begin{array}{l}124 \\
130 \\
140 \\
125 \\
132\end{array}$ & $\begin{array}{r}1270 \\
1389 \\
626 \\
239 \\
254\end{array}$ & $\begin{array}{l}0.75 \\
1.17 \\
1.80 \\
2.55 \\
3.85\end{array}$ & $\begin{array}{l}0.95 \\
1.16 \\
2.09 \\
6.55 \\
8.39\end{array}$ & $\begin{array}{l}119 \\
113 \\
105 \\
125 \\
120\end{array}$ & $\begin{array}{l}122 \\
122 \\
123 \\
125 \\
126\end{array}$ \\
\hline $\begin{array}{c}184 \\
2-3 \\
195 \\
173 \\
81\end{array}$ & $\begin{array}{r}19 \\
11 \\
7 \\
15 \\
12\end{array}$ & $\begin{array}{l}\mathbf{M} \\
\mathbf{M} \\
\mathbf{M} \\
\mathbf{M} \\
\mathbf{M}\end{array}$ & \begin{tabular}{l|}
69 \\
58 \\
44.5 \\
64.5 \\
56
\end{tabular} & $\begin{array}{r}129 \\
88 \\
42 \\
147 \\
71\end{array}$ & $\begin{array}{r}11-0 \\
1-6 \\
1-4 \\
3-0 \\
5-0\end{array}$ & $\begin{array}{l}28.4 \\
23.0 \\
26.0 \\
27.9 \\
34.3\end{array}$ & $\begin{array}{r}2987 \\
334 \\
3452 \\
2070 \\
3618\end{array}$ & $\begin{array}{l}0.52 \\
5.10 \\
0.20 \\
0.88 \\
0.38\end{array}$ & $\begin{array}{l}0.50 \\
6.99 \\
0.43 \\
0.99 \\
0.54\end{array}$ & $\begin{array}{l}138 \\
136 \\
161 \\
137 \\
144\end{array}$ & $\begin{array}{r}1747 \\
397 \\
472 \\
588 \\
2816\end{array}$ & $\begin{array}{l}1.02 \\
3.73 \\
1.83 \\
3.94 \\
0.42\end{array}$ & $\begin{array}{l}0.99 \\
5.11 \\
3.97 \\
4.40 \\
0.61\end{array}$ & $\begin{array}{r}113 \\
117 \\
96 \\
124 \\
119\end{array}$ & $\begin{array}{l}126 \\
127 \\
129 \\
131 \\
132\end{array}$ \\
\hline $\begin{array}{r}65 \\
86 \\
109 \\
79 \\
106\end{array}$ & $\begin{array}{r}15 \\
14 \\
6 \\
12 \\
11\end{array}$ & $\begin{array}{c}\mathbf{M} \\
\mathbf{M} \\
\mathbf{F} \\
\mathbf{M} \\
\mathbf{M}\end{array}$ & $\begin{array}{l}60 \\
62 \\
44 \\
54 \\
55\end{array}$ & $\begin{array}{l}93 \\
91 \\
44 \\
78 \\
78\end{array}$ & $\begin{array}{l}3-4 \\
4-8 \\
5-4 \\
4-0 \\
4-7\end{array}$ & $\begin{array}{l}19.1 \\
27.9 \\
17.3 \\
26.2 \\
24.1\end{array}$ & $\begin{array}{r}477 \\
2457 \\
1711 \\
1936 \\
2373\end{array}$ & $\begin{array}{l}3.40 \\
0.71 \\
0.34 \\
0.80 \\
0.51\end{array}$ & $\begin{array}{l}4.39 \\
0.87 \\
0.73 \\
1.24 \\
0.76\end{array}$ & $\begin{array}{l}146 \\
152 \\
157 \\
152 \\
159\end{array}$ & $\begin{array}{r}240 \\
1126 \\
338 \\
526 \\
318\end{array}$ & $\begin{array}{l}5.50 \\
1.80 \\
2.12 \\
3.02 \\
4.56\end{array}$ & $\begin{array}{l}7.10 \\
2.20 \\
4.56 \\
4.68 \\
6.84\end{array}$ & $\begin{array}{l}119 \\
119 \\
119 \\
125 \\
120\end{array}$ & $\begin{array}{l}133 \\
136 \\
138 \\
139 \\
140\end{array}$ \\
\hline $\begin{array}{l}180 \\
192 \\
150\end{array}$ & $\begin{array}{r}10 \\
13 \\
7\end{array}$ & $\begin{array}{l}\mathbf{M} \\
\mathbf{M} \\
\mathbf{M}\end{array}$ & $\begin{array}{l}51 \\
56 \\
48\end{array}$ & $\begin{array}{l}67 \\
73 \\
51\end{array}$ & $\begin{array}{l}5-0 \\
4-6 \\
2-0\end{array}$ & $\begin{array}{l}22.4 \\
29.0 \\
32.7\end{array}$ & $\begin{array}{r}566 \\
2703 \\
610\end{array}$ & $\begin{array}{l}3.32 \\
0.59 \\
4.58\end{array}$ & $\begin{array}{l}5.58 \\
0.87 \\
8.47\end{array}$ & $\begin{array}{l}188 \\
161 \\
211\end{array}$ & $\begin{array}{r}287 \\
2800 \\
547\end{array}$ & $\begin{array}{l}4.17 \\
0.51 \\
2.95\end{array}$ & $\begin{array}{l}7.01 \\
0.75 \\
5.46\end{array}$ & $\begin{array}{l}120 \\
155 \\
122\end{array}$ & $\begin{array}{l}154 \\
158 \\
167\end{array}$ \\
\hline
\end{tabular}

not similar to ours since they apparently reported the incidence of determinations rather than, as we did, the number of children examined. They also gave urea preliminary to the test in order to insure maximum clearance. However, their results are in agreement with our conclusions.

Holt (4) called attention to the greater variability of the augmentation limits in children and suggested that this might be a serious limitation to the usefulness of the test in children. The present data indicate that, for children over 4 years, this suggested limitation does not hold. We have not studied children under 4 years of age but Schoenthal, Lurie and Kelly (5) reported that in 9 normal infants urea clearance values corresponded to those of normal adults.

It would appear, therefore, that the blood urea clearance test of renal function is as applicable to the study of renal damage in children as it is in adults and that the range and distribution of values are similar.

The group with a history of acute hematuric nephritis. The data for these children are given in Table II and summarized to show the distribution of clearance values in the lower histogram of Figure 1.

It is evident at once that the second purpose of this study, namely, to determine whether there is any evidence of consistently occurring residual damage that is detectable by the clearance test in children with a history of hematuric nephritis, is answered in an unequivocal manner. That is to say, the mean and distribution of the test values of this group are indistinguishable from those of 
the control group. (See Figure 1.) It is especially significant that the value for standard deviation of this group, like that of the control group, indicates a "stable universe."

While it seems clear that in this group as a whole the ability of the kidneys to excrete urea is essentially normal there were several children who had moderately low clearance values and who merit special consideration. See below under "Clinical Discussion." that one month may be arbitrarily selected as the time when the immediate effect of the acute stage had ceased. The data were accordingly divided on the basis of intervals since cessation of acute symptoms of 1 month to 1 year; 1 to 5 years; and 5 years or more. It appears (Table III) that there is no significant variation between these sub-groups. The mean for the sub-group " 1 month to 1 year" of 104.2 is somewhat less than the mean for the whole group but the difference

TABLE III

Biometric analysis of urea clearance tests

\begin{tabular}{|c|c|c|c|c|c|c|}
\hline \multicolumn{4}{|c|}{ History of acute hematuric nephritis } & \multicolumn{3}{|c|}{ No evidence of kidney damage } \\
\hline & $\begin{array}{l}\text { Sub- } \\
\text { jects }\end{array}$ & Mean & $\begin{array}{l}\text { Standard } \\
\text { deviation }\end{array}$ & $\begin{array}{l}\text { Sub- } \\
\text { jects }\end{array}$ & Mean & $\begin{array}{l}\text { Standard } \\
\text { deviation }\end{array}$ \\
\hline Entire group & 78 & $\begin{array}{l}\text { per cent normal } \\
109.1 \pm 1.5\end{array}$ & $19.2 \pm 1.0$ & 62 & $\begin{array}{c}\text { per cent normal } \\
107.3 \pm 1.4\end{array}$ & $15.9 \pm 1.0$ \\
\hline $\begin{array}{c}\text { Age, years } \\
4-8 \\
8-12 \\
12-16 \\
16+\end{array}$ & $\begin{array}{r}16 \\
33 \\
24 \\
6\end{array}$ & $\begin{array}{r}116.9 \pm 3.3 \\
106.1 \pm 2.0 \\
111.5 \pm 2.4 \\
96.7 \pm 6.1\end{array}$ & $\begin{array}{l}19.7 \pm 2.3 \\
17.3 \pm 1.4 \\
17.3 \pm 1.7 \\
22.2 \pm 4.3\end{array}$ & $\begin{array}{l}14 \\
35 \\
13\end{array}$ & $\begin{array}{l}107.9 \pm 2.4 \\
106.2 \pm 1.7 \\
109.4 \pm 3.7\end{array}$ & $\begin{array}{l}13.2 \pm 1.7 \\
15.1 \pm 1.2 \\
20.0 \pm 2.6\end{array}$ \\
\hline $\begin{array}{l}\text { Boys } \\
\text { Girls }\end{array}$ & $\begin{array}{l}45 \\
33\end{array}$ & $\begin{array}{l}113.5 \pm 2.0 \\
103.1 \pm 2.0\end{array}$ & $\begin{array}{l}19.6 \pm 1.4 \\
17.0 \pm 1.4\end{array}$ & $\begin{array}{l}42 \\
20\end{array}$ & $\begin{array}{l}108.1 \pm 1.7 \\
105.5 \pm 2.2\end{array}$ & $\begin{array}{l}16.5 \pm 1.2 \\
14.4 \pm 1.5\end{array}$ \\
\hline \multirow{2}{*}{$\begin{array}{l}\mathrm{CmCm} \\
\mathrm{CsCm} \\
\mathrm{CsCs}_{\mathrm{sC}} \\
\mathrm{Cm} \\
\mathrm{Cs}\end{array}$} & $\begin{array}{r}52 \\
15 \\
9\end{array}$ & $\begin{array}{l}104.7 \pm 1.7 \\
117.8 \pm 3.3 \\
122.5 \pm 3.4\end{array}$ & $\begin{array}{l}18.2 \pm 1.2 \\
18.7 \pm 2.3 \\
15.3 \pm 2.4\end{array}$ & $\begin{array}{l}47 \\
14\end{array}$ & $\begin{array}{l}107.5 \pm 1.5 \\
106.1 \pm 3.0\end{array}$ & $\begin{array}{l}15.7 \pm 1.1 \\
16.5 \pm 2.1\end{array}$ \\
\hline & & & & $\begin{array}{l}47 \\
15\end{array}$ & $\begin{array}{l}112.1 \pm 2.0 \\
116.2 \pm 2.5\end{array}$ & $\begin{array}{l}19.9 \pm 1.4 \\
14.4 \pm 1.8\end{array}$ \\
\hline $\begin{array}{l}\text { T.S.A.S.* } \\
1-12 \text { months } \\
1-5 \text { years } \\
5+\end{array}$ & $\begin{array}{l}12 \\
57 \\
15\end{array}$ & $\begin{array}{l}104.2 \pm 3.1 \\
108.3 \pm 1.8 \\
112.5 \pm 3.5\end{array}$ & $\begin{array}{l}16.0 \pm 2.2 \\
20.3 \pm 1.3 \\
19.9 \pm 2.5\end{array}$ & & & \\
\hline \multicolumn{4}{|c|}{$\begin{array}{l}\text { Activity and cardiac lesions } \\
\text { Bed (includes } 6 \text { cardiacs) } \\
\text { Bath and dining (includes } 1 \text { cardiac) } \\
\text { All heart cases } \\
\text { Limited and full (includes } 6 \text { cardiacs) } \\
\text { Limited and full (No cardiacs) }\end{array}$} & $\begin{array}{l}12 \\
12 \\
13 \\
38 \\
32\end{array}$ & $\begin{array}{l}105.0 \pm 3.7 \\
113.8 \pm 2.8 \\
111.3 \pm 3.0 \\
105.9 \pm 1.6 \\
105.8 \pm 1.9\end{array}$ & $\begin{array}{l}19.0 \pm 2.6 \\
14.3 \pm 2.0 \\
15.8 \pm 2.1 \\
14.8 \pm 1.1 \\
16.0 \pm 1.3\end{array}$ \\
\hline
\end{tabular}

* Time since acute symptoms.

Although the distribution histogram of this data, which parallels that of the control group, suggests that analysis of sub-groups could show only minor differences, some of the analyses are interesting because of the very lack of influence of factors often considered important.

One of the first questions was that of the influence of the time interval since cessation of acute symptoms (Table II). From the data of the " acute group," Table V, it may be concluded is not significant. It would, however, be definitely incorrect to conclude that all traces of acute damage have always entirely disappeared within one month after cessation of the acute symptoms. Case 5, for example, showed at three months a definitely lowered clearance which became normal after $3 \frac{1}{2}$ months.

At first glance there would appear to be a somewhat greater influence of age on the clearance in this post-nephritic group than in the con- 
trol group, but more careful study shows that there is no mathematically significant difference between the mean for the age sub-groups from four to sixteen years. The number of children in the sub-group " 16 years or more" is so small (6) that no significance can be attributed to its low value.

The difference between mean values for boys and for girls might suggest a sex factor for this group. However, the low mean value for the girls is not significantly different from the means of the total of either major group. Moreover the difference between the mean values of the boys and of the girls is not significant by biometric criteria. That is to say, in order for the difference of the two means to be significant the value of the difference of the means divided by the probable error of the difference

$$
\left(K=\frac{\text { Difference }}{\text { Probable error difference }}\right)
$$

should be greater than four (odds 142 to 1 ), whereas the difference between the means of boys and girls in this instance is only 3.71. Stating this in another way, the odds are only 80 to 1 that this difference between the means of boys and girls is not due to chance alone.

It is an interesting coincidence that the coefficients of variation of urea clearance of our cases in the two major groups are about 17 which is close to the coefficient of variation of the size of the healthy kidney, 16.8 (6). The authors do not take this to mean that variations in urea clearance are wholly dependent on variation in size of kidneys.

In both groups of children phenolsulphonphthalein tests were made coincidently with the clearance tests. The comparison of the two tests for each child showed so little correlation that it does not seem worth while to tabulate them. This lack of correlation may be due to the fact that the dye

TABLE IV

Additional data on those children of the group with a history of hematuric nephritis who had either a relatively low clearance or other symptoms of kidney abnormality

\begin{tabular}{|c|c|c|c|c|c|c|c|c|c|c|c|c|c|c|}
\hline \multirow{2}{*}{$\begin{array}{l}\text { Subject } \\
\text { number }\end{array}$} & \multirow{2}{*}{ Age } & \multirow{2}{*}{ Sex } & \multirow{2}{*}{\multicolumn{2}{|c|}{ T.S.A.S.† }} & \multirow{2}{*}{$\begin{array}{c}\text { Blood } \\
\text { pressure }\end{array}$} & \multirow{2}{*}{ Urine } & \multirow{2}{*}{$\begin{array}{c}\text { Blood } \\
\text { urea } \\
\text { nitrogen }\end{array}$} & \multicolumn{3}{|c|}{ Urea clearance } & \multicolumn{3}{|c|}{ Phenolsulphonpthalein' } & \multirow{2}{*}{$\begin{array}{c}\text { Concentra- } \\
\text { tion test }\end{array}$} \\
\hline & & & & & & & & $\begin{array}{c}\text { 1st } \\
\text { period }\end{array}$ & $\underset{\text { period }}{\text { 2nd }}$ & $\begin{array}{c}\text { Aver- } \\
\text { age }\end{array}$ & $\begin{array}{c}\text { 1st } \\
\text { hour }\end{array}$ & $\begin{array}{l}\text { 2nd } \\
\text { hour }\end{array}$ & Total & \\
\hline \multirow[b]{2}{*}{104} & \multirow{2}{*}{$\begin{array}{c}\text { years } \\
18\end{array}$} & \multirow[b]{2}{*}{$\mathbf{F}$} & \multicolumn{2}{|c|}{ years-months } & \multirow{2}{*}{$\begin{array}{l}m m . H g \\
106 / 76 \\
124 / 84\end{array}$} & \multirow[b]{2}{*}{ alb. +} & \multirow{2}{*}{$\begin{array}{c}\text { mgm. per } \\
100 \text { cc. } \\
10 \\
12\end{array}$} & \multirow{2}{*}{$\begin{array}{c}\text { per } \\
\text { cent } \\
55 \\
59\end{array}$} & \multirow{2}{*}{$\begin{array}{r}\text { per } \\
\text { cent } \\
56 \\
61\end{array}$} & \multirow{2}{*}{$\begin{array}{r}\text { per } \\
\text { cent } \\
56 \\
60\end{array}$} & \multirow{2}{*}{$\begin{array}{c}\text { per } \\
\text { cent } \\
39 \\
34\end{array}$} & \multirow{2}{*}{$\begin{array}{c}\text { per } \\
\text { cent } \\
22 \\
29\end{array}$} & \multirow{2}{*}{$\begin{array}{c}\text { per } \\
\text { cent } \\
61 \\
63\end{array}$} & \multirow[t]{2}{*}{$\begin{array}{l}\text { specific } \\
\text { gravity }\end{array}$} \\
\hline & & & $\begin{array}{l}2 \\
3\end{array}$ & $\begin{array}{l}3 \\
5\end{array}$ & & & & & & & & & & \\
\hline 187 & 7 & $\mathbf{M}$ & 2 & 7 & $98 / 66$ & $\mathbf{0}$ & 15 & 87 & 64 & 76 & 53 & 13 & 66 & \\
\hline 80 & 18 & F & $\begin{array}{l}6 \\
7\end{array}$ & & $\begin{array}{l}118 / 78 \\
114 / 78\end{array}$ & $\begin{array}{l}\mathbf{0} \\
\mathbf{0}\end{array}$ & $\begin{array}{r}11 \\
8\end{array}$ & $\begin{array}{l}79 \\
84\end{array}$ & $\begin{array}{l}73 \\
72\end{array}$ & $\begin{array}{l}76 \\
78\end{array}$ & $\begin{array}{l}46 \\
53\end{array}$ & $\begin{array}{l}41 \\
19\end{array}$ & $\begin{array}{l}87 \\
72\end{array}$ & \\
\hline 188 & 10 & $\mathbf{F}$ & 3 & 4 & $110 / 68$ & $\mathbf{0}$ & 10 & 79 & 76 & 78 & 31 & 28 & 59 & \\
\hline 101 & 8 & $\mathbf{F}$ & 1 & & $108 / 70$ & Tr. alb. & 6 & 80 & 76 & 78 & 23 & 23 & 46 & \\
\hline 88 & 12 & F & & $\begin{array}{r}6 \\
6 \\
10\end{array}$ & $120 / 90$ & alb. ++ & $\begin{array}{r}6 \\
10 \\
9\end{array}$ & $\begin{array}{l}73 \\
78 \\
89\end{array}$ & $\begin{array}{l}71 \\
79 \\
76\end{array}$ & $\begin{array}{l}72 \\
79 \\
83\end{array}$ & $\begin{array}{l}39 \\
56 \\
64\end{array}$ & $\begin{array}{r}24 \\
22 \\
9\end{array}$ & $\begin{array}{l}63 \\
78 \\
73\end{array}$ & $1.027^{*}$ \\
\hline 179 & 14 & $\mathbf{M}$ & $\begin{array}{l}6 \\
6\end{array}$ & $\begin{array}{l}4 \\
8\end{array}$ & $\begin{array}{l}130 / 74 \\
111 / 68\end{array}$ & $\underset{0}{\text { alb }_{j}}+$ & $\begin{array}{l}13 \\
15\end{array}$ & $\begin{array}{r}141 \\
98\end{array}$ & $\begin{array}{l}100 \\
102\end{array}$ & $\begin{array}{l}121 \\
100\end{array}$ & $\begin{array}{l}34 \\
67\end{array}$ & $\begin{array}{l}28 \\
10\end{array}$ & $\begin{array}{l}62 \\
77\end{array}$ & $1.031^{*}$ \\
\hline 69 & 13 & $\mathbf{M}$ & $\begin{array}{l}4 \\
4 \\
5\end{array}$ & $\begin{array}{l}9 \\
6\end{array}$ & $\begin{array}{l}158 / 100 \\
130 / 84 \\
120 / 90\end{array}$ & $\begin{array}{l}\text { alb. }++ \\
\text { alb. }++ \\
\text { alb. }+\end{array}$ & $\begin{array}{l}10 \\
11 \\
12\end{array}$ & $\begin{array}{r}108 \\
112 \\
96\end{array}$ & $\begin{array}{l}128 \\
106 \\
111\end{array}$ & $\begin{array}{l}118 \\
109 \\
104\end{array}$ & $\begin{array}{l}44 \\
20 \\
61\end{array}$ & $\begin{array}{l}27 \\
30 \\
10\end{array}$ & $\begin{array}{l}71 \\
60 \\
71\end{array}$ & $\begin{array}{l}1.024 \oplus \\
1.020^{*}\end{array}$ \\
\hline 5 & 14 & F & 1 & $\begin{array}{l}3 \\
3 \frac{1}{2} \\
8\end{array}$ & $\begin{array}{l}110 / 80 \\
110 / 70\end{array}$ & $\begin{array}{c}\mathbf{0} \\
\mathbf{0} \\
\text { alb. }+\end{array}$ & $\begin{array}{l}10 \\
13 \\
12\end{array}$ & $\begin{array}{r}38 \\
115 \\
119\end{array}$ & $\begin{array}{l}60 \\
134 \\
100\end{array}$ & $\begin{array}{r}49 \\
125 \\
110\end{array}$ & 57 & 19 & 76 & \\
\hline
\end{tabular}

† Time since acute symptoms.

Corrected to hourly basis.

* Lashmet and Newburgh, (9).

$\oplus$ Mosenthal (10). 
was injected subcutaneously, and the statement may not be true for results of intravenous phenolsulphonphthalein injections or of the fractional test (Shaw (7), Chapman and Halsted (8)).

Clinical discussion. Additional data are recorded in Table IV concerning six children who had clearance values below 85 (first six cases of Table II) or had evidence suggestive of kidney disease despite the normal clearance value. (See Cases 179, 69 and 5.)

Three of the children (Cases 80, 187 and 188) had no symptoms suggestive of kidney damage.

It should be noted that in five of these six children with relatively low clearance there was a comparatively small difference between the successive hourly clearance values (exception 187). This in itself is probably without significance but if associated with continuously low or decreasing clearance values, it may possibly be considered evidence of loss of flexibility of kidney function.

The urine of three children (Cases 104, 101 and 88) contained albumin. In one of these three (Case 88) the albuminuria was of the orthostatic type and the kidney was able to concentrate the urine to a specific gravity of 1.027 .

The last three children listed in Table IV (179, 69 and 5) had clearance values well within the normal range but had albuminuria on at least one examination. In only one (Case 69) is there general evidence for a diagnosis of chronic nephritis. He had had a constant albuminuria with few or no casts in the urinary sediment. His systolic blood pressure ranged from 120 to 160 ; it has been as high as 140 since the last examination noted in Table IV. In addition his kidneys were unable to concentrate urine to a specific gravity of more than 1.020. While he had had no manifest edema, on several occasions there were fluctuations in weight of 6 to 8 pounds.

None of the children listed in this table is incapacitated. It is planned to follow the future progress of all of them.

DETERMINATION OF UREA CLEARANCE VALUES OF PATIENTS WITH ACUTE HEMATURIC NEPHRITIS

In Table $\mathrm{V}$ are given the data for the children who were studied during the acute stage of hematuric nephritis. All but two (Cases 152 and 161) were studied also during and after convalescence.
Five cases are included $(5,59,121,160,164)$ for which there are no data during the acute stage.

The manner in which the kidneys recover from the acute attack is shown clearly in this table. It is apparent that, in general, the clearance value has usually returned to the normal zone within one month, although there are exceptions (see Cases 5 and 160). In Case 5 the clearance was only 49 three months after acute symptoms had ceased, but after four months the clearance was normal. In Case 74 there was a stormy course in the hospital with a second acute attack following rheumatic infection three months after admission to the hospital.

The data for this group demonstrate the usefulness of the clearance test for determining the extent of initial damage to the kidneys and for ascertaining when the function of the kidney has been restored to normal.

\section{SUM MARY}

1. The blood urea clearance values were determined in a group of 62 children who had no evidence of kidney disease. The results are analysed biometrically. The distribution and mean values corresponded to those of normal adults.

2. Similar studies were made in a group of 78 children with a history of acute hematuric nephritis. The distribution and means of the urea clearance values coincided with those from the group in which there was no history of kidney damage. There is evidence that most of these children had no residual kidney damage due to their acute condition.

3. Data are given for the clearance values during the acute stage of, and convalescence from, hematuric nephritis. In the majority of cases the kidney function had returned to normal within one month after cessation of the acute symptoms.

4. The two types of clearance values, "maximum" and "standard," appeared to be entirely comparable.

\section{BIBLIOGRAPHY}

1. Möller, E., McIntosh, J. F., and Van Slyke, D. D., Studies of urea excretion. II. Relationship between urine volume and the rate of urea excretion by normal adults. J. Clin. Invest., 1928, 6, 427.

2. McIntosh, J. F., Möller, E., and Van Slyke, D. D., Ibid. III. The influence of body size on urea output. J. Clin. Invest., 1928, 6, 467. 
TABLE V

Urea clearance values in children during the acute stage of, and convalescence from, hematuric nephritis

\begin{tabular}{|c|c|c|c|c|c|c|c|c|c|c|c|c|c|c|c|c|}
\hline \multirow{3}{*}{$\begin{array}{l}\text { Subject } \\
\text { number }\end{array}$} & \multirow{3}{*}{ Age } & \multirow{3}{*}{ Sex } & \multirow{3}{*}{ Height } & \multirow{3}{*}{ Weight } & \multirow{3}{*}{$\begin{array}{l}\text { Time } \\
\text { since } \\
\text { onset }\end{array}$} & \multirow{3}{*}{ T.S.A.S.† } & \multirow{3}{*}{$\begin{array}{c}\text { Blood } \\
\text { urea }\end{array}$} & \multicolumn{4}{|c|}{ 1st period } & \multicolumn{4}{|c|}{ 2nd period } & \multirow{3}{*}{$\underset{\text { (av.) }}{C}$} \\
\hline & & & & & & & & \multicolumn{3}{|c|}{ Urine } & \multirow{2}{*}{$C_{1}$} & \multicolumn{3}{|c|}{ Urine } & \multirow{2}{*}{$C_{2}$} & \\
\hline & & & & & & & & Urea & V & $\mathrm{V}_{\text {cor }}$ & & Urea & V & $V_{\text {cor. }}$. & & \\
\hline $\begin{array}{r}74-1 \\
2 \\
3 \\
4 \\
5 \\
6 \\
7 \\
8 \\
9 \\
10\end{array}$ & 7 & $\mathbf{M}$ & $\begin{array}{l}51 \\
51\end{array}$ & $\begin{array}{l}58 \\
61.5 \\
69.5 \\
61 \\
58 \\
56 \\
55 \\
56\end{array}$ & 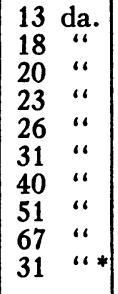 & 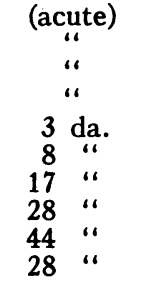 & $\begin{array}{r}216 \\
111.8 \\
54.7 \\
39.1 \\
28.3 \\
30.4 \\
31.2 \\
26.2 \\
27.3 \\
28.7\end{array}$ & $\begin{array}{r}711 \\
608 \\
711 \\
811 \\
795 \\
821 \\
1127 \\
1829 \\
573 \\
665\end{array}$ & $\begin{array}{l}0.09 \\
1.64 \\
0.20 \\
0.66 \\
0.70 \\
1.08 \\
0.32 \\
0.37 \\
2.38 \\
2.67\end{array}$ & $\begin{array}{l}0.15 \\
2.77 \\
0.34 \\
1.12 \\
1.18 \\
1.83 \\
0.53 \\
0.62 \\
4.24 \\
4.51\end{array}$ & $\begin{array}{r}2 \\
20 \\
14 \\
41 \\
57 \\
68 \\
49 \\
102 \\
119 \\
139\end{array}$ & $\begin{array}{c}25 \text { hou } \\
456 \\
617 \\
639 \\
611 \\
731 \\
903 \\
784 \\
242 \\
737\end{array}$ & $\begin{array}{l}\text { ur spec } \\
2.10 \\
1.37 \\
1.04 \\
1.39 \\
1.50 \\
1.40 \\
2.14 \\
5.00 \\
2.25\end{array}$ & $\begin{array}{l}\text { imen } \\
3.55 \\
2.32 \\
1.76 \\
2.35 \\
2.54 \\
2.37 \\
3.62 \\
8.90 \\
3.78\end{array}$ & $\begin{array}{r}2 \\
19 \\
35 \\
40 \\
68 \\
80 \\
92 \\
144 \\
105 \\
129\end{array}$ & $\begin{array}{r}2 \\
20 \\
25 \\
41 \\
63 \\
74 \\
71 \\
123 \\
112 \\
134\end{array}$ \\
\hline $\begin{array}{r}75-1 \\
2 \\
3 \\
4 \\
5 \\
6\end{array}$ & 8 & $\mathbf{M}$ & $\begin{array}{l}51.5 \\
52 \\
53\end{array}$ & 58 & $\begin{array}{cc}18 & \text { da. } \\
21 & ، \\
26 & ، \\
35 & ، \\
47 & ، \\
13 & \text { mo. }\end{array}$ & $\begin{array}{l}\text { (acute) } \\
2 \text { da. } \\
7 \\
16 ، \\
28 ، \\
12 \text { mo. }\end{array}$ & $\begin{array}{l}19.9 \\
27.1 \\
23.9 \\
25.0 \\
24.6 \\
20.1\end{array}$ & $\begin{array}{r}270 \\
541 \\
653 \\
335 \\
1810 \\
215\end{array}$ & $\begin{array}{l}1.60 \\
1.23 \\
1.31 \\
2.40 \\
0.63 \\
4.42\end{array}$ & $\begin{array}{l}2.67 \\
2.05 \\
2.19 \\
3.98 \\
1.05 \\
6.94\end{array}$ & $\begin{array}{r}48 \\
55 \\
80 \\
71 \\
139 \\
99\end{array}$ & $\begin{array}{l}220 \\
268 \\
538 \\
286 \\
238 \\
200\end{array}$ & $\begin{array}{l}2.60 \\
2.44 \\
1.44 \\
3.55 \\
3.22 \\
4.22\end{array}$ & $\begin{array}{l}4.34 \\
4.07 \\
2.40 \\
5.89 \\
5.35 \\
6.63\end{array}$ & $\begin{array}{l}64 \\
54 \\
72 \\
90 \\
69 \\
88\end{array}$ & $\begin{array}{r}56 \\
55 \\
76 \\
81 \\
104 \\
94\end{array}$ \\
\hline $\begin{array}{r}162-1 \\
2 \\
3 \\
4\end{array}$ & 8 & $F$ & 54 & & $\begin{array}{rr}5 & \text { da. } \\
11 & ، \\
18 & ، \\
25 & ،\end{array}$ & $\begin{array}{c}\text { (acute) } \\
\dddot{،} \\
6 \mathrm{da} .\end{array}$ & $\begin{array}{c}120.1 \\
28.5 \\
51 \\
41.7\end{array}$ & $\begin{array}{r}1050 \\
709 \\
873 \\
1621\end{array}$ & $\begin{array}{l}0.39 \\
0.61 \\
0.33 \\
0.46\end{array}$ & $\begin{array}{l}0.59 \\
0.94 \\
0.51 \\
0.71\end{array}$ & $\begin{array}{l}13 \\
44 \\
23 \\
61\end{array}$ & $\begin{array}{r}1201 \\
253 \\
715 \\
557\end{array}$ & $\begin{array}{l}0.43 \\
2.60 \\
1.42 \\
2.42\end{array}$ & $\begin{array}{l}0.66 \\
4.00 \\
2.19 \\
3.73\end{array}$ & $\begin{array}{l}15 \\
47 \\
41 \\
67\end{array}$ & $\begin{array}{l}14 \\
46 \\
32 \\
64\end{array}$ \\
\hline 159 & 12 & $\mathbf{M}$ & 59 & 78 & $10 \mathrm{da}$. & (acute) & 23.6 & 246 & 2.63 & 3.50 & 49 & 252 & 2.57 & 3.42 & 49 & 49 \\
\hline $\begin{array}{r}198-1 \\
2\end{array}$ & 9 & $\mathrm{~F}$ & $\begin{array}{l}53 \\
53.5\end{array}$ & $\begin{array}{l}54 \\
67\end{array}$ & $\begin{array}{r}13 \mathrm{da} . \\
5 \mathrm{mo} .\end{array}$ & $\begin{array}{c}\text { (acute) } \\
4 \frac{1}{2} \mathrm{mo} \text {. }\end{array}$ & $\begin{array}{l}43 \\
30.8\end{array}$ & $\begin{array}{l}1388 \\
3005\end{array}$ & $\begin{array}{l}0.35 \\
0.37\end{array}$ & $\begin{array}{l}0.55 \\
0.59\end{array}$ & $\begin{array}{r}44 \\
139\end{array}$ & $\begin{array}{r}693 \\
1012\end{array}$ & $\begin{array}{l}1.97 \\
1.24\end{array}$ & $\begin{array}{l}3.09 \\
1.95\end{array}$ & $\begin{array}{l}66 \\
85\end{array}$ & $\begin{array}{r}55 \\
112\end{array}$ \\
\hline 161 & 7 & $\mathrm{~F}$ & 47 & 42 & $13 \mathrm{da}$. & (acute) & 27.6 & 1162 & 0.19 & 0.38 & 48 & 530 & 0.73 & 1.44 & 43 & 46 \\
\hline 152 & 5 & $\mathbf{M}$ & 41 & & $14 \mathrm{da}$. & (acute) & 37.1 & & & & & 140 & 3.55 & 8.38 & 42 & 42 \\
\hline $\begin{array}{r}170-1 \\
2\end{array}$ & 5 & $\mathbf{M}$ & 45.5 & & $\begin{array}{ll}14 \\
21\end{array}$ & $\begin{array}{c}\text { (acute) } \\
1 \text { da. }\end{array}$ & $\begin{array}{l}31.5 \\
25\end{array}$ & $\begin{array}{r}217 \\
1286\end{array}$ & $\begin{array}{l}1.12 \\
0.41\end{array}$ & $\begin{array}{l}2.28 \\
0.84\end{array}$ & $\begin{array}{l}21 \\
87\end{array}$ & $\begin{array}{r}180 \\
1277\end{array}$ & $\begin{array}{l}0.49 \\
0.35\end{array}$ & $\begin{array}{l}1.01 \\
0.72\end{array}$ & $\begin{array}{l}11 \\
80\end{array}$ & $\begin{array}{l}16 \\
84\end{array}$ \\
\hline $\begin{array}{r}114-1 \\
2\end{array}$ & 7.5 & $\mathrm{~F}$ & 50.5 & $\begin{array}{l}43 \\
46\end{array}$ & 24 da. & $\begin{array}{c}\text { (acute) } \\
35-40 \text { da. }\end{array}$ & $\begin{array}{l}47 \\
30.5\end{array}$ & $\begin{array}{r}490 \\
1609\end{array}$ & $\begin{array}{l}3.50 \\
0.62\end{array}$ & $\begin{array}{l}5.92 \\
1.06\end{array}$ & $\begin{array}{r}82 \\
100\end{array}$ & $\begin{array}{l}396 \\
284\end{array}$ & $\begin{array}{l}2.83 \\
3.24\end{array}$ & $\begin{array}{l}4.78 \\
5.54\end{array}$ & $\begin{array}{l}53 \\
69\end{array}$ & $\begin{array}{l}68 \\
85\end{array}$ \\
\hline $\begin{array}{r}121-1 \\
2\end{array}$ & 8 & $\mathbf{M}$ & 48.5 & 56 & $\begin{array}{r}68 \mathrm{da} . \\
3 \mathrm{mo} .\end{array}$ & $\begin{array}{l}30 \mathrm{da} . \\
2 \mathrm{mo} .\end{array}$ & $\begin{array}{l}23.9 \\
20.8\end{array}$ & $\begin{array}{r}1236 \\
340\end{array}$ & $\begin{array}{l}0.35 \\
2.62\end{array}$ & $\begin{array}{l}0.63 \\
4.82\end{array}$ & $\begin{array}{r}76 \\
105\end{array}$ & $\begin{array}{r}1030 \\
243\end{array}$ & $\begin{array}{l}1.07 \\
4.42\end{array}$ & $\begin{array}{l}1.93 \\
8.13\end{array}$ & $\begin{array}{l}111 \\
127\end{array}$ & $\begin{array}{r}94 \\
116\end{array}$ \\
\hline 164 & 2.5 & $\mathrm{~F}$ & 36 & & $11 \mathrm{da}$. & 5 da. & 15.5 & 556 & 1.15 & 3.22 & 154 & 158 & 2.79 & 7.81 & 106 & 130 \\
\hline $\begin{array}{r}59-1 \\
2\end{array}$ & 9 & $\mathbf{M}$ & $\begin{array}{l}52 \\
53\end{array}$ & $\begin{array}{l}53 \\
57\end{array}$ & $\begin{array}{c}29 \text { da. } \\
7 \frac{1}{2} \mathrm{mo} .\end{array}$ & $\begin{array}{r}15 \mathrm{da} . \\
7 \mathrm{mo} .\end{array}$ & $\begin{array}{l}25 \\
30.2\end{array}$ & $\begin{array}{l}509 \\
360\end{array}$ & $\begin{array}{l}2.16 \\
3.96\end{array}$ & $\begin{array}{l}3.50 \\
6.26\end{array}$ & $\begin{array}{r}95 \\
100\end{array}$ & $\begin{array}{l}625 \\
633\end{array}$ & $\begin{array}{l}1.33 \\
1.93\end{array}$ & $\begin{array}{l}2.15 \\
3.05\end{array}$ & $\begin{array}{l}72 \\
85\end{array}$ & $\begin{array}{l}84 \\
93\end{array}$ \\
\hline 160 & 14 & F & 62 & 94 & $54 \mathrm{da}$. & 39 da. & 19.3 & 773 & 0.17 & 0.20 & 33 & 408 & 1.78 & 2.16 & 61 & 47 \\
\hline $\begin{array}{r}5-1 \\
2 \\
3\end{array}$ & 12 & $\mathbf{M}$ & $\begin{array}{l}57.5 \\
57.5 \\
60.5\end{array}$ & $\begin{array}{r}95 \\
97 \\
107\end{array}$ & $\mid \begin{array}{cc}4 & \text { mo. } \\
4 \frac{1}{2} & \text { ، } \\
21 & \text {. }\end{array}$ & $\begin{array}{l}3 \text { mo. } \\
3 \frac{1}{2} “ ، \\
20\end{array}$ & $\begin{array}{l}21 \\
27 \\
24.9\end{array}$ & $\begin{array}{r}256 \\
1557 \\
1345\end{array}$ & $\begin{array}{l}1.65 \\
0.82 \\
1.11\end{array}$ & $\begin{array}{l}2.31 \\
1.15 \\
1.41\end{array}$ & $\begin{array}{r}38 \\
115 \\
119\end{array}$ & $\begin{array}{l}512 \\
410 \\
375\end{array}$ & $\begin{array}{l}1.23 \\
4.73 \\
3.93\end{array}$ & $\begin{array}{l}1.72 \\
6.62 \\
4.99\end{array}$ & $\begin{array}{r}59 \\
134 \\
100\end{array}$ & $\begin{array}{r}49 \\
125 \\
110\end{array}$ \\
\hline
\end{tabular}


3. Payne, W. W., and Shukry, H., The urea clearance test in children. Arch. Dis. Childhood, 1934, 9, 335.

4. Holt, L. Emmett, Jr., Urea clearance tests. Their limitations as a measure of renal function in childhood. Am. J. Dis. Child., 1932, 44, 306.

5. Schoenthal, Ludwig, Lurie, Dorothy, and Kelly, Margaret, Urea clearance in normal and in dehydrated infants. Renal function in intestinal intoxication. Am. J. Dis. Child., 1933, 45, 41.

6. Pearl, Raymond, Introduction to Medical Biometry and Statistics. W. B. Saunders Company, Philadelphia, 1930, 2d ed., p. 347. Quoted from Greenmund.
7. Shaw, E. C., A study of the curve of elimination of phenolsulphonphthalein by normal and diseased kidneys. J. Urol., 1925, 13, 575.

8. Chapman, E. M., and Halsted, J. A., The fractional phenolsulphonphthalein test in Bright's disease. Am. J. M. Sc., 1933, 186, 223.

9. Lashmet, F. H., and Newburgh, L. H., The specific gravity of the urine as a test of kidney function. J. A. M. A., 1930, 94, 1883.

10. Mosenthal, H. O., Renal function as measured by the elimination of fluids, salt and nitrogen and the specific gravity of the urine. Arch. Int. Med., 1915, 16, 733. 